\title{
une méthode pour le dimensionnement à la rupture des ouvrages en sols renforcés
}

\author{
a method for the yield design of reinforced soil structures
}

\author{
A. ANTHOINE \\ Chargée de recherche au C.N.R.S. \\ Laboratoire de Mécanique des Solides \\ Ecole Polytechnique
}

Rev. Franç. Géotech. n 50, pp. 5-17 (janvier 1990)

\section{Résumé}

On présente une méthode de calcul pour les ouvrages de soutènement en sols renforcés (terre armée, clouage, géotextile). Fondée sur la théorie du calcul à la rupture, cette méthode est à la fois mécaniquement rigoureuse et numériquement rapide. En matière de sécurité, elle s'inspire du calcul aux états limites pour le béton armé. Cette philosophie, nouvelle pour les ouvrages de soutènement, est particulièrement bien adaptée au caractère composite du sol renforcé.

La méthode a été mise en œuvre sur micro-ordinateur : la structure du logiciel STARS (mode conversationnel) permet d'exploiter facilement et systématiquement toutes les informations fournies.

\section{Abstract}

A design method for reinforced soil retaining structures (reinforced earth, nailing, geotextile) is presented. Based upon the yield design theory, it is both mechanically rigorous and numerically rapid. As regards safety considerations, it is derived from the limit state design for reinforced concrete. This way of thinking is new as far as retaining structures are concerned and is well adapted to the composite character of reinforced soils.

The method has been implemented on a personal computer : the structure of the program STARS (conversational mode) makes it possible to derive easily the maximum advantage of the results. 


\section{INTRODUCTION}

Il existe actuellement de nombreuses méthodes de calcul quí permettent d'analyser la stabilité des ouvrages en sol renforcé. Elles peuvent être réparties en trois grandes catégories :

- celles qui s'inspirent des différentes méthodes qui ont été développées à l'origine pour les ouvrages non renforcés (Fellenius, Bishop, perturbations, etc.) en y introduisant et prenant en compte les efforts résistants dus au renforcement de diverses façons [BLONDEAU et al., 1984], [DELMAS et al., 1986], [LESHCHINSKY et al., 1985]. Ces méthodes, relativement rapides, ne sont pas mécaniquement rigoureuses et peuvent, dans certains cas, conduire à des sur- ou sous-estimations importantes des capacités réelles de louvrage ;

- celles qui sont de type «éléments finis " parmi lesquelles il faut distinguer celles qui font appel à des lois de comportement [JURAN et al., 1985) et celles qui sont basées sur la théorie des charges limites [PASTOR et al., 1986]. Ces méthodes sont mécaniquement rigoureuses mais en général très coûteuses numériquement. En effet, la taille des renforcements impose souvent un maillage très fin sur l'ensemble de la structure ;

- celles qui sont fondées sur la théorie du calcul à la rupture dans le cadre de l'homogénéisation des milieux périodiques [de BUHAN et al., 1987]. Ces méthodes sont à la fois rapides et mécaniquement rigoureuses mais elles sont restreintes au cas des renforcements périodiques de période suffisamment petite comparée aux dimensions de l'ouvrage étudié, ce qui est rarement le cas.

La méthode présentée dans cet article conjugue rigueur mécanique, rapidité de calcul et souplesse d'utilisation. Elle permet d'étudier la stabilité des pentes, remblais et fouilles renforcés par des inclusions unidimensionnelles (clouage, terre armée) ou bidimensionnelles (géotextile).

Dans le paragraphe 2 , le principe de la méthode fondée sur la théorie du calcul à la rupture, est présenté en termes concrets. Cet exposé comprend la liste des hypothèses de calcul, la définition du coefficient caractérisant la stabilité de l'ouvrage (facteur de confiance) ainsi que la manière dont ce coefficient peut être calculé.

Dans le paragraphe 3 , la méthode est comparée de manière systématique aux méthodes les plus courantes, c'est-à-dire celles de la première des trois catégories citées antérieurement.

Le paragraphe 4 est exclusivement consacré à la manière dont la sécurité est prise en compte; il s'agit d'une conception nouvelle pour les ouvrages de soutènement, conforme aux réflexions actuellement menées pour les recommandations relatives au clouage des sols.

Cette méthode a été mise en ceuvre sur microordinateur: le paragraphe 5 est en quelque sorte la notice d'utilisation du logiciel obtenu (STARS, version de juin 89) comprenant la description des entrées, des sorties, du support informatique requis et du mode de fonctionnement.

Enfin, dans le paragraphe 6 sont présentés deux exemples d'utilisation du programme. Le premier montre comment exploiter de manière systématique les résultats fournis. Le second est un exemple réaliste de dimensionnement.

\section{PRINCIPE GÉNÉRAL DE LA MÉTHODE}

Cette méthode de calcul relève rigoureusement de la théorie du calcul à la rupture [SALENÇON, 1983] et peut être présentée dans le formalisme mathématique correspondant [ANTHOINE, 1989]. Toutefois. nous avons délibérément choisi de la présenter ici en des termes familiers au mécanicien des sols, termes dont la signification exacte sera parfois reprécisée.

\subsection{Position du problème}

Considérons l'ouvrage renforcé représenté en plan sur la figure 1. Du point de vue du calcul à la rupture, il est caractérisé par :

- sa géométrie (forme du talus et schéma de renforcement) ;

- son mode de chargement (poids propre et surcharges) ;

- ses capacités de résistance (sol, inclusions et interfaces sol-inclusion).

Les données qui définissent la géométrie et le mode de chargement ne présentent pas d'ambiguïté alors que celles relatives aux capacités de résistance doivent être explicitées :

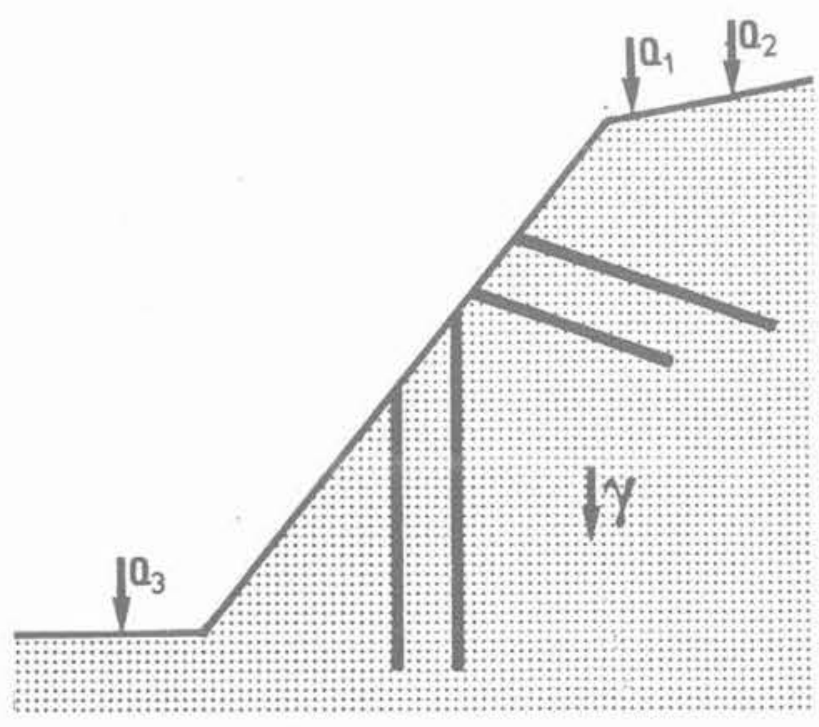

Fig. 1. - Un exemple de talus renforce.

Fig. 1. - The example of a reinforced embankment. 
- la résistance du sol est caractérisée par un critère de Coulomb (cohésion $\mathrm{C}$ et angle de frottement interne $\phi$ ) ;

- les inclusions résistent en traction (effort normal maximal $N_{0}$ ) et, éventuellement en compression (effort normal minimal $-\lambda N_{\circ}$ avec $0 \leq \lambda \leq 1$ ). Toute résistance en flexion et en cisaillement est ici négligée :

- l'interface sol-inclusion est de type Tresca. Elle est caractérisée par le frottement latéral $\mathrm{f}_{1}$ qui est défini comme l'effort d'arrachement limite pour un mètre linéaire d'inclusion.

S'agissant d'un problème plan, les quantités $N_{0}$ et $f_{1}$ sont rapportées au mètre linéaire transversal d'ouvrage.

Toutes les données de géométrie, de chargement et de résistance étant connues a priori, le problème est alors de savoir si l'ouvrage est stable et, dans l'affirmative, de quantifier la marge de sécurité qu'il présente.

\subsection{Définition du facteur de confiance $\Gamma$}

Pour que louvrage soit stable, il faut en particulier que l'équilibre global de toutes ses parties soit assuré compte tenu des capacités de résistance limitées des constituants. Considérons, par exemple, un volume $V$ limité par le terrain naturel et un arc $A B$ de spirale logarithmique de foyer $\Omega$ et d'angle $\phi$ (fig. 2).

Pour étudier l'équilibre global de ce volume, il faut faire le bilan des forces qui lui sont appliquées. Cellesci se répartissent en deux groupes :

- les forces actives : parmi les forces composant le chargement de l'ouvrage, ce sont celles qui s'appliquent effectivement au volume $\mathrm{V}$. Ce sont donc toutes des forces connues a priori. Pour l'exemple considéré sur la figure 2 , il s'agit du poids propre du bloc et des forces linéiques $Q_{1}$ et $Q_{3}$;

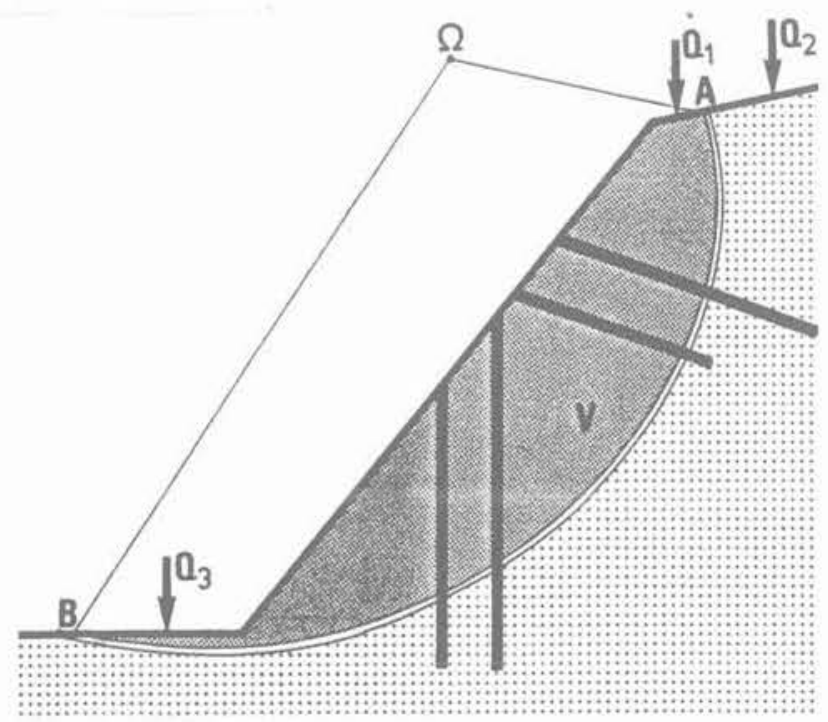

Fig. 2. - Volume de sol limité par une spirale logarithmique d'angle $\phi$.

Fig. 2. - Volume of soil limited by a logspiral of angle $\phi$.
- les efforts passifs : ce sont les efforts développés par les constituants que rencontre la frontière $A B$ du volume $\mathrm{V}$ (contrainte $\mathrm{I}=(\sigma, \tau)$ dans le sol, effort normal $\mathrm{N}$ dans les inclusions). Ces efforts ne sont donc pas connus a priori mais ils doivent respecter les critères de résistance correspondants (critère de Coulomb pour le sol, effort normal maximal pour les barres, frottement latéral à l'interface).

Pour que le volume $\mathrm{V}$ soit en équilibre, il faut qu'il existe une répartition d'efforts passifs le long de sa frontière $A B$, qui contrebalance l'effet des forces actives. En particulier, le moment résultant des forces actives $M_{\text {act }}$, et celui des efforts passifs, tous deux calculés par rapport à $\Omega$ mais avec des conventions de signes opposées, doivent être égaux. Comme les efforts passifs sont limités par les différents critères de résistance, leur moment résultant l'est aussi par une valeur que l'on notera $M_{\text {rés }}$ et que l'on appellera moment résistant maximal. Ainsi, le rapport $\Gamma_{\mathrm{V}}=$ $\mathrm{M}_{\mathrm{rés}} / \mathrm{M}_{\mathrm{act}}$ vérifie les propriétés suivantes :

$\Gamma_{V}<1 \Leftrightarrow$ l'équilibre global du volume $\mathrm{V}$ n'est pas assuré.

$\Gamma_{V} \geq 1 \Leftrightarrow$ l'équilibre global du volume $V$ pourra être assuré en moment.

Si $\Gamma$ désigne la valeur minimale atteinte par $\Gamma_{V}$ lorsque le volume $\mathrm{V}$ varie, alors :

$\Gamma<1 \Leftrightarrow$ louvrage n'est pas stable : l'équilibre giobal d'au moins un volume $V$ n'est pas assuré.

$\Gamma \geq 1 \Leftrightarrow$ louvrage est stable: l'équilibre global de tous les volumes $V$ pourra être assuré en moment.

De plus, le volume $V$ pour lequel $\Gamma_{V}$ est minimal sera dit critique. $\Gamma$ sera appelé facteur de confiance : il constitue une mesure de la marge de sécurité (si $\Gamma \geq 1$ ) ou d'insécurité (si $\Gamma<1$ ) de l'ouvrage. En principe, la qualité de l'information donnée par $\Gamma$ peut être améliorée de deux manières:

- soit en testant l'équilibre global de chaque volume $\mathrm{V}$ non seulement en moment mais aussi en résultante ;

- soit en considérant d'autres volumes que ceux limités par un arc de spirale logarithmique d'angle $\phi$.

En pratique, ces deux approches, qui pourraient d'ailleurs être cumulées, sont numériquement coûteuses et la plupart du temps inefficaces dans la mesure où elles améliorent rarement la précision du facteur de confiance $\Gamma$ [SALENÇON, 1989]. En particulier, nous verrons plus loin que, dans un sol frottant $(\phi \neq 0)$, l'équilibre d'un volume limité par un arc de cercle de centre $\Omega$, est toujours assuré en moment par rapport à $\Omega$ et ceci quelles que soient les forces actives. Autrement dit, pour un tel volume, $\Gamma_{\mathrm{V}}$ est infini.

\subsection{Calcul du facteur de confiance $\Gamma$}

$\Gamma$ est la valeur minimale du rapport $\Gamma_{V}=M_{r e s s} / M_{\text {act }}$ lorsque le volume $\mathrm{V}$ varie. Pour un volume $\mathrm{V}$ donné, le calcul de $\mathrm{M}_{\text {act }}$, moment résultant des forces actives, ne présente pas de difficultés particulières puis- 
que toutes ces forces sont connues a priori. En revan-

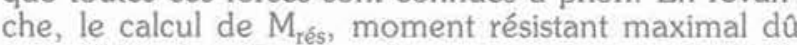
aux efforts passifs, découle, comme son nom l'indique, d'une maximisation. En effet, ces efforts ne sont pas connus mais seulement astreints à respecter les capacités de résistance des constituants.

Pour calculer $M_{\text {ress, examinons les efforts qui peuvent }}$ se développer le long de l'arc de spirale logarithmique $A B$ qui limite le volume $V$ (fig. 3). II faut distinguer les efforts passifs dus au sol (contrainte $\mathrm{T}=(\sigma, \tau)$ ) et ceux dus aux inclusions (effort normal $\mathrm{N}$ ).

La loupe 1 montre ce qui se passe dans le sol, en un point $M$ de la frontière $A B$ du volume $V$. Une propriété de la spirale logarithmique d'angle $\phi$ est que la normale extérieure au volume $\underline{\mathrm{n}}$ fait un angle $\phi$ avec le rayon vecteur $\Omega \mathrm{M}$. La contrainte qui s'applique en $M$ sur la facette de normale $n$ est représen- tée par un vecteur $I$ d'origine $M$. Pour que le critère de résistance du sol soit respecté, l'extrémité du vecteur $\mathrm{I}$ doit rester à l'intérieur du cône de Coulomb représenté dans le repère $(M, n, t)$. Il s'ensuit que le moment de $\mathrm{I}$ par rapport à $\Omega$ est au plus égal à $\Omega \mathrm{M} \times \mathrm{C} \cos \phi$, cette valeur étant par définition le moment résistant maximal que peut fournir le sol au point M. Pour obtenir cette valeur, il n'a pas été nécessaire de déterminer $\underline{T}$ et on remarque d'ailleurs, qu'il existe une infinité de vecteurs I conduisant au même résultat.

Les loupes 2, 4 et 5 montrent, dans trois configurations différentes, ce qui se passe au niveau d'une inclusion interceptée par la frontière $A B$. La force qui s'applique en $M$ sur la partie de l'inclusion appartenant au volume, est égale à $\mathrm{Nm}$ où $\mathrm{N}$ désigne l'effort normal dans l'inclusion (compté positivement en traction) et $\mathrm{m}$ le vecteur directeur de l'inclusion, dirigé

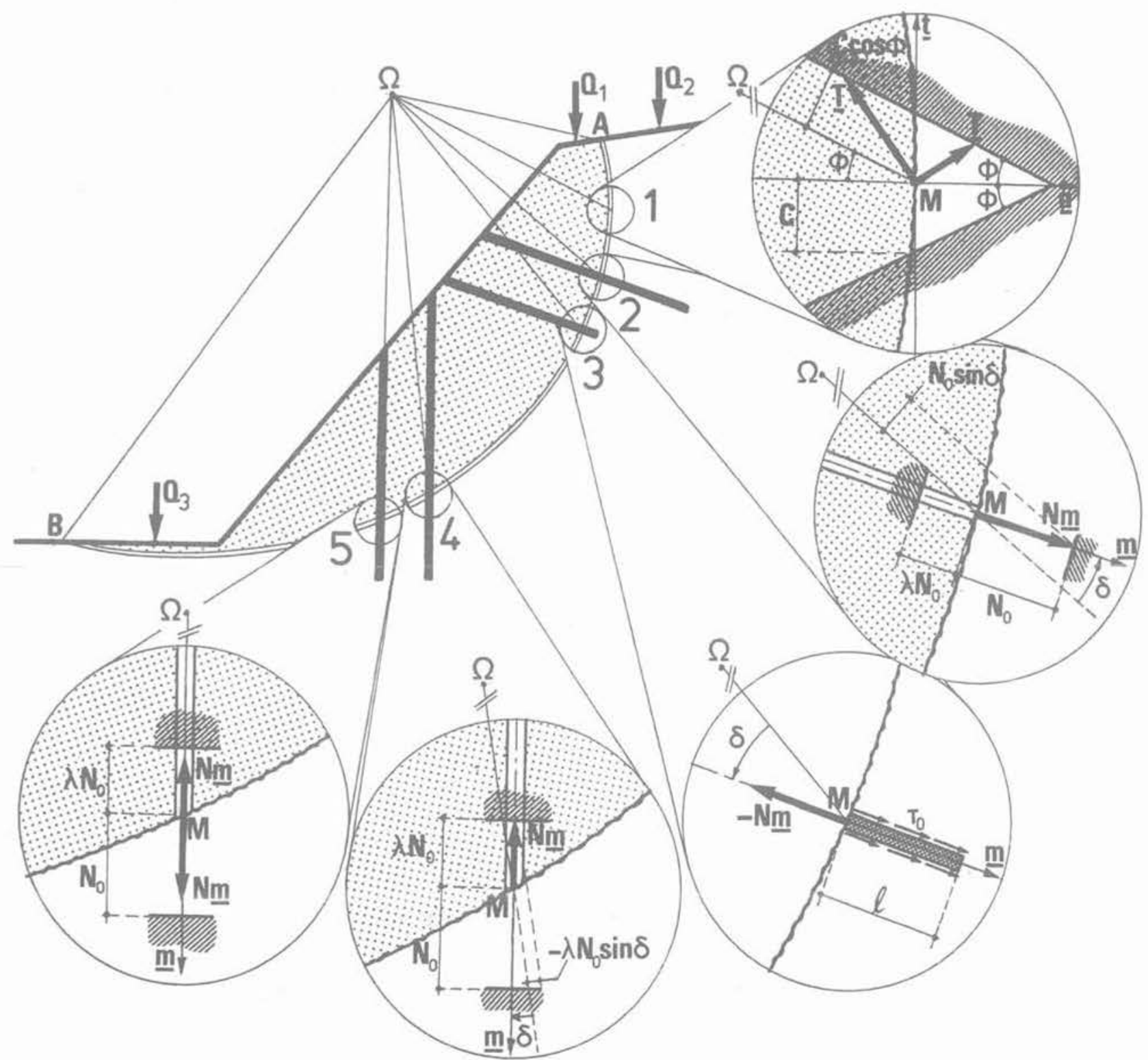

Fig. 3. - Calcul du moment résistant maximal: contribution des efforts développés le long de la frontière $A B$.

Fig. 3. - Computation of the maximum resisting moment: contribution of the forces developed along the boundary AB. 
vers l'extérieur du volume. Le moment de cette force par rapport à $\Omega$ est égal à $\Omega \mathrm{M} \times \mathrm{N}$ sin $\delta$ si $\delta$ désigne l'angle que fait $m$ avec le rayon vecteur $\Omega \mathrm{M}$. Pour que le critère de l'inclusion soit respecté, il faut que l'effort normal $N$ reste compris entre $-\lambda N_{0}$ et $N_{0}$. La valeur maximale du moment dû à $\mathrm{N}$ va donc dépendre du signe de $\delta$ :

- si $\delta>0$ (loupe 2), le moment résistant maximal vaut $\Omega \mathrm{M} \times \mathrm{N}_{0} \sin \delta$ car il est obtenu pour $\mathrm{N}=\mathrm{N}_{\mathrm{o}}$, l'inclusion étant alors sollicitée en traction:

- si $\delta=0$ (loupe 4), quelle que soit la valeur de $\mathrm{N}$, son moment est toujours nul;

- si $\delta<0$ (loupe 5), le moment résistant maximal vaut $-\Omega \mathrm{M} \times \lambda \mathrm{N}_{\mathrm{o}} \sin \delta$ car il est obtenu pour $\mathrm{N}=$ $-\lambda N_{0}$, l'inclusion étant alors sollicitée en compression.

Dans les trois cas, le moment résistant maximal est donné par $\Omega \mathrm{M} \times \sup \left(\mathrm{N}_{\mathrm{o}} \sin \delta ;-\lambda \mathrm{N}_{\mathrm{o}} \sin \delta\right)$. On remarque que le mode de sollicitation de l'inclusion (traction ou compression) ne dépend que de la position de l'inclusion par rapport au foyer $\Omega$ de la spirale (angle $\delta$ ).

La loupe 3 montre enfin ce qui se passe à l'interface sol-inclusion. Pour que la portion d'inclusion de longueur $\ell$ qui se trouve à l'extérieur du volume, soit en équilibre, il faut que l'effort $-\mathrm{Nm}$ qui lui est appliqué au point $M$ puisse être effectivement équilibré par la résultante des efforts de cisaillement $\tau$ à l'interface sur la longueur extérieure $\ell$. Ces derniers étant limités par le critère de Tresca, la norme de l'effort normal $\mathrm{N}$ ne peut en aucun cas excéder la valeur $\mathrm{lf}_{1}$. Le critère d'interface impose donc sur le moment résistant fourni par l'effort normal $\mathrm{N}$, une limitation qui peut, dans certains cas, s'avérer plus restrictive que le propre critère de résistance de l'inclusion. La valeur maximale du moment résistant calculée précédemment doit alors être révisée à la baisse :

- si $\delta \geq 0$ et $\ell f_{1}<N_{0}$, on obtient $\Omega \mathrm{M} \times \mathrm{lf}_{1} \sin \delta$, ce qui correspond à l'arrachement de l'inclusion sollicitée en traction ;

- si $\delta \leq 0$ et $\ell f_{1}<\lambda N_{0}$, on obtient $-\Omega M \times \ell_{1} \sin \delta$, ce qui correspond à l'enfoncement de l'inclusion sollicitée en compression.

Finalement, le moment résistant maximal total $M_{\text {rés, }}$, résulte de la somme des contributions dues au sol et aux inclusions. $M_{\text {tés }}$ est donc égal à l'intégrale du terme $\Omega \mathrm{M} \times \mathrm{C} \cos \phi$ lorsque le point $\mathrm{M}$ décrit l'arc de spirale $A B$, augmentée du terme $\Omega M \times \sup \left\{\inf \left(N_{0}\right.\right.$; $\left.\left.\ell f_{1}\right) \sin \delta ;-\inf \left(\lambda N_{0} ; \ell f_{1}\right) \sin \delta\right\}$ chaque fois que la spirale intercepte une inclusion. Le calcul de $M_{\text {rés }}$ est donc très simple, d'autant plus que l'intégrale le long de la spirale possède une expression analytique explicite.

Le raisonnement qui vient d'être détaillé pour un volume limité par une spirale logarithmique d'angle $\phi$ peut également être appliqué à un volume $V$ limité par une droite (fig. 4). A la place des moments $M_{\text {act }}$ et $M_{\text {rés }}$, on considère les forces résultantes $R_{\text {act }}$ et $R_{\text {rés }}$ suivant la direction inclinée d'un angle $\phi$ par rapport à la droite frontière $\mathrm{AB}$. En examinant successivement les efforts passifs dus au sol et aux inclusions, on montre que la résultante résistante maximale $R_{\text {rés }}$ est

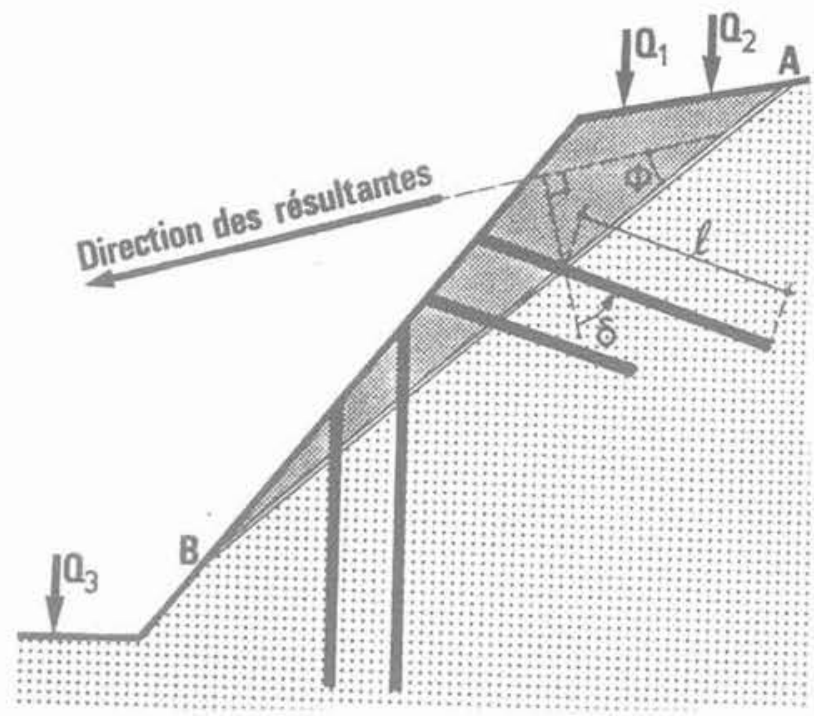

Fig. 4. - Volume de sol limité par une droite.

Fig. 4. - Volume of soil limited by a straight line.

égale à l'intégrale le long de la ligne de rupture de la quantité $\mathrm{C} \cos \phi$, augmentée du terme sup (inf( $\mathrm{N}_{0}$; $\left.\left.\ell \mathrm{f}_{1}\right) \sin \delta ;-\inf \left(\lambda \mathrm{N}_{0} ; \mathrm{Pf}_{1}\right) \sin \delta\right\}$ chaque fois que la ligne de rupture rencontre une inclusion ( $\delta$ et $\hat{l}$ sont indiqués sur la figure 4). Le rapport $\Gamma_{V}=R_{\text {rés }} / R_{\text {act }}$ est le majorant correspondant du facteur de confiance $\Gamma$ de l'ouvrage. Ce résultat s'obtient directement à partir des moments $M_{a c t}$ et $M_{\text {rés }}$ en considérant un volume limité par une spirale logarithmique d'angle $\phi$ dont le foyer serait rejeté à l'infini.

\section{COMPARAISON AVEC D'AUTRES MÉTHODES}

Nous nous référons ici aux méthodes de calcul les plus courantes (Fellenius, Bishop, Spencer, perturbations, etc.) qui sont toutes basées sur le même principe: l'étude de la stabilité de certains volumes de sol sous. l'action conjuguée des forces actives (ou motrices) et des efforts passif́s (ou résistants). Les seuls points sur lequels ces méthodes sont susceptibles de différer fondamentalement sont

- la courbe frontière des volumes testés (cercle, ligne brisée, etc.) :

- la manière dont le moment résistant maximal (ou la résultante résistante maximale) est calculé, le calcul du moment actif (ou de la résultante active) ne présentant aucune difficulté.

Toutes ces méthodes sont basées sur un certain nombre d'hypothèses arbitraires qui permettent de déterminer, le long de la frontière du volume, une distribution d'efforts passifs supposée fournir le moment maximal (ou la résultante maximale). Ces hypothèses varient d'une méthode à l'autre. Citons entre autres :

- le découpage du volume en tranches verticales ;

- lannulation des cisaillements entre deux tranches ;

- la courbe de variation des efforts le long de la frontière ;

- le cône de diffusion des forces linéiques ; 
- l'angle critique à partir duquel une inclusion est sollicitée en traction ou en compression.

Le point fort de la méthode présentée est qu'elle permet de calculer le moment résistant maximal $M_{\text {rés }}$ (ou la résultante $\mathrm{R}_{\text {rés }}$ ) sans recourir à la détermination d'une distribution d'efforts passifs le long de la frontière du volume. Elle présente donc un double avantage par rapport aux autres méthodes :

- elle est mécaniquement rigoureuse car elle ne fait appel à aucune hypothèse arbitraire. Seules sont nécessaires les données minimales, c'est-à-dire la géométrie de l'ouvrage, son chargement et les capacités de résistance de ses constituants ;

- elle est plus rapide car $M_{\text {rés }}$ (ou $R_{\text {rés }}$ ) est calculé directement à partir de la forme de la frontière et des critères de résistance des constituants rencontrés (voir $\S 2.3$.$) . En particulier, il n'est pas nécessaire de$ découper le volume en tranches.

Il faut enfin signaler que le choix d'une frontière en forme de spirale logarithmique d'angle $\phi$ n'est pas arbitraire. En effet, quelle que soit la frontière considérée (arc de cercle, ligne brisée, etc.), il est toujours possible et aisé de calculer le moment résistant maximal par rapport à un point quelconque (ou la résultante résistante maximale suivant une direction quelconque) ainsì que le moment résultant (ou la résultante) des forces actives correspondant. On obtient bien à chaque fois un nouveau majorant $\Gamma_{V}=$ $\mathrm{M}_{\text {rés }} / \mathrm{M}_{\text {act }}$ (ou $\mathrm{R}_{\text {rés }} / \mathrm{R}_{\text {act }}$ ) du facteur de confiance $\Gamma$ de l'ouvrage mais l'expérience montre que, s'il n'est pas infini, il n'améliore quasiment jamais l'estimation obtenue à partir des seules spirales d'angle $\phi$. Considérons par exemple, la forme de frontiètre la plus couramment utilisée, un arc de cercle $A B$ de centre $\Omega$ (fig. 5) et calculons le moment résistant maximal $M_{\text {rés }}$ par rapport à $\Omega$ de la même manière qu'au paragraphe 2.3.

Le calcul des contributions dues aux inclusions n'est pas fondamentalement modifié dans la mesure où l'expression $\Omega \mathrm{Msup}\left(\inf \left(\mathrm{N}_{0} ; \ell \mathrm{f}_{1}\right) \sin \delta ;-\inf \left(\lambda \mathrm{N}_{0}\right.\right.$; $\left.\mathrm{\ell f}_{1}\right) \sin \delta$ ] est encore valable. En revanche, le moment

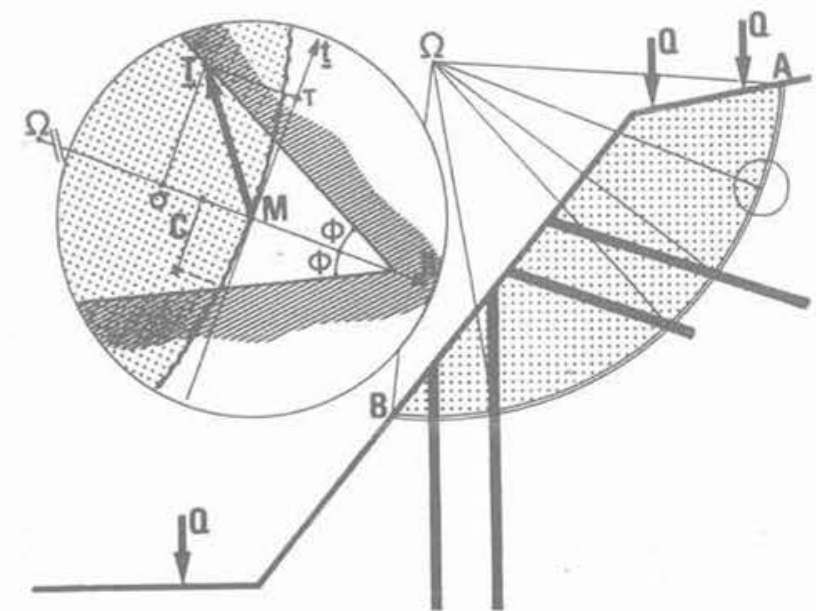

Fig. 5. - Calcul du moment résistant maximal dû au sol pour un volume limité par un arc de cercle $A B$.

Fig. 5. - Computation of the maximum resisting moment due to the soil for a volume limited by an arc of a circle $A B$. résistant maximal dû au sol est infini. Pour le montrer, examinons à nouveau ce qui ce passe dans le sol en un point $M$ de l'arc $A B$ (loupe de la figure 5). Cette fois-ci, la normale extérieure $n$ au bloc est portée par le rayon vecteur $\Omega \mathrm{M}$ si bien que le moment par rapport à $\Omega$ du vecteur contrainte $I=(\sigma, \tau)$ est égal à $\tau \times \Omega \mathrm{M}$. Bien que l'extrémité du vecteur $\mathrm{T}$ doive rester à l'intérieur du cône de Coulomb, Ta quantité $\tau \times \Omega \mathrm{M}$ n'est pas bornée: il suffit, par exemple, de choisir $\mathrm{I}=(-\tau / \operatorname{tg} \phi, \tau)$ et de faire tendre $\tau$ vers $+\infty$ pour obtenir un moment infini. Par conséquent, le moment résistant maximal total $\mathrm{M}_{\text {rés }}$ est infini ainsi que le rapport $\Gamma_{V}=M_{r e s s} / M_{\text {act }}$ et ceci, quelle que soit la valeur de $M_{\text {act }}$. Nous venons ainsi de montrer que, dans un sol frottant $(\phi \neq 0)$, l'équilibre d'un volume limité par un arc de cercle de centre $\Omega$ est toujours assuré en moment par rapport à $\Omega$ quelles que soient les forces actives appliquées. Dans les méthodes de calcul courantes, ce résultat est faussé : des hypothèses arbitraires supplémentaires permettent de déterminer une valeur particulière du vecteur contrainte $T$, si bien que le moment résistant correspondant est toujours fini. Le seul cas où la méthode de calcul présentée et les méthodes courantes peuvent se rejoindre est obtenu lorsque langle de frottement $\phi$ est nul (une spirale logarithmique d'angle nul est un cercle).

\section{APPROCHE DE LA SÉCURITÉ}

En matière de sécurité, la philosophie de la méthode présentée s'inspire du calcul aux états limites pour le béton armé (codes BAEL et CEB). Cette approche de la sécurité, nouvelle pour les ouvrages de soutènement, est conforme aux réflexions menées par les recommandations CLOUTERRE actuellement en cours de rédaction.

\subsection{Coefficients de sécurité partiels}

En plus des données définissant la géométrie, les capacités de résistance et le mode de chargement de l'ouvrage, il est possible d'imposer des coefficients de sécurité partiels de deux types:

- ceux relatifs aux capacités de résistance des constituants (sol, inclusion, interface sol-inclusion) : chaque caractéristique de résistance $\left(\phi, C, N_{0}, f_{1}\right)$ est pondérée par un réel supérieur à l'unité ( $\mathrm{F} \phi, \mathrm{F}_{\mathrm{C}}$, $\mathrm{F}_{\mathrm{N}_{0}}, \mathrm{~F}_{\mathrm{f}_{1}}$ ) de manière à ce que les critères obtenus soient inclus dans les critères initiaux ( $\phi^{\prime}$ tel que tg $\phi$ $=\operatorname{tg} \phi^{\prime} / \mathrm{F} \phi, \mathrm{C}^{\prime}=\mathrm{C} / \mathrm{F}_{\mathrm{C}}, \mathrm{N}_{\mathrm{o}}^{\prime}=\mathrm{N}_{\mathrm{o}} / \mathrm{F}_{\mathrm{N}_{0}}, \mathrm{f}_{1}=$ $\left.f_{1} / F_{f_{1}}\right)$. En particulier, pour que le critère de Coulomb vérifie cette propriété, la condition $F_{\phi} \leq F_{C}$ est nécessaire. Tout en généralisant l'égalité $F_{\phi}=F_{C}$ couramment utilisée, cette inégalité traduit le fait que la cohésion d'un sol est généralement moins bien connue que son angle de frottement, rejoignant en cela les réglementations canadienne et danoise ;

- ceux relatifs au chargement (poids propre et surcharges) : à chaque charge sont affectés deux coefficients, l'un minorateur ( $\mathrm{F}^{-} \leq 1$ ), l'autre majorateur $\left(\mathrm{F}^{+} \geq 1\right.$ ), car il n'est pas toujours possible de savoir a priori si une charge est ou non stabilisatrice. Toute 
charge $\mathrm{Q}$ pourra donc varier entre $\mathrm{QF}_{\mathrm{Q}}{ }^{-}$et $\mathrm{QF}_{\mathrm{Q}^{+}}{ }^{+}$. Seul le poids propre $\gamma$ fait exception et sera seulement majoré.

D'une manière générale, les valeurs caractéristiques des capacités de résistance et des charges sont définies de manière probabiliste et les coefficients de sécurité associés sont liés aux probabilités d'occurrence et de dépassement.

\subsection{Définition du facteur de confiance $\Gamma$ compte tenu de la sécurité}

Les coefficients de sécurité partiels sont respectés si l'ouvrage est encore stable dans la configuration la plus pessimiste c'est-à-dire pour les capacités de résistance pondérées donc minimales et pour le chargement le plus défavorable (charges majorées ou minorées suivant le cas). Le facteur de confiance $\Gamma$ vérifie alors les propriétés suivantes:

$\Gamma<1 \Leftrightarrow$ l'ouvrage n'est pas stable dans la configuration la plus pessimiste : l'équilibre global d'au moins un volume $\mathrm{V}$ ne peut pas être assuré compte tenu de la sécurité exigée ;

$\Gamma \geq 1 \Leftrightarrow$ l'ouvrage est stable dans la configuration la plus pessimiste : l'équilibre global de tous les volumes $\mathrm{V}$ testés pourra être assuré en moment compte tenu de la sécurité exigée.

Ainsi défini, le facteur de confiance $\Gamma$ mesure la marge de sécurité (ou d'insécurité) qui subsiste une fois que tous les coefficients partiels ont été pris en compte. Le concepteur devra donc faire en sorte que $\Gamma$ soit aussi proche que possible de l'unité par valeurs supérieures. En pratique, la valeur minimale de $\Gamma$ poura être légèrement supérieure à l'unité (1,05 par exemple) afin de tenir compte de lincertitude due aux calculs.

\subsection{Calcul du facteur de confiance $\Gamma$ compte tenu de la sécurité}

C'est la définition même du facteur de confiance qui dicte la manière dont les coefficients de sécurité partiels doivent être introduits dans le calcul de $\Gamma$. Le facteur de confiance est toujours la valeur minimale du rapport $\Gamma_{V}=M_{\text {rés }} / M_{\text {act }}$ lorsque le volume $\mathrm{V}$ varie. Simplement, les capacités de résistance et les charges doivent être pondérées de manière à ce que, d'une part, le moment résistant maximal $\mathrm{M}_{\text {rés }}$ soit le plus faible possible et que, d'autre part, le moment résultant des forces actives $M_{a c t}$ soit le plus fort possible.

La prise en compte des coefficients de sécurité partiels sur les capacités de résistance est très facile à mettre en œuvre: il suffit, avant tout calcul, de remplacer les caractéristiques nominales de résistance par leurs valeurs pondérées. En particulier, les volumes considérés doivent être limités par des spirales logarithmiques d'angle $\phi$ '.

En revanche, la prise en compte des coefficients de sécurité partiels sur les charges ne peut pas être mise en œuvre de manière aussi systématique : une même charge pouvant se révéler stabilisatrice pour un volume et déstabilisatrice pour un autre, sa pondération (minoration ou majoration) doit être révisée à chaque nouveau calcul de $M_{\text {act. }}$. Ainsi une charge active est majorée (resp. minorée) si son moment est positif (resp. négatif) c'est-à-dire si elle est défavorable (resp. favorable) pour le volume considéré. Le volume $\mathrm{V}$ pour lequel le rapport $\Gamma_{V}$ est minimal sera encore appelé volume critique et une charge sera déclarée favorable ou défavorable pour l'ouvrage si, et seulement si, elle lest pour le volume critique.

\subsection{Comparaison avec l'approche usuelle de la sécurité}

Il s'agit ici de comparer le facteur de confiance $\Gamma$ qui vient d'être défini, au coefficient de sécurité $F$ traditionnellement utilisé pour les ouvrages de soutènement.

On rappelle la définition de ce coefficient $F$ : soit $V$ un volume limité par le terrain naturel et une frontière quelconque. Le coefficient de sécurité $F_{V}$ du volume $\mathrm{V}$, supposé constant le long de la frontière, est le rapport de la contrainte de cisaillement maximale admissible dans le sol, à la contrainte de cisaillement mobilisée le long de la frontière. Cela revient à considérer que le volume $\mathrm{V}$ est à l'k équilibre limite ", c'est-à-dire que le moment actif est égal au moment résistant maximal avec les caractéristiques réduites $\mathrm{C} / \mathrm{F}_{\mathrm{V}}$ et $\operatorname{arctg}\left(\operatorname{tg} \phi / \mathrm{F}_{\mathrm{V}}\right)$. Le coefficient de sécurité $\mathrm{F}$ de l'ouvrage est alors la valeur minimale de $F_{\mathrm{V}}$ lorsque le volume $\mathrm{V}$ varie.

Il est donc clair que le coefficient de sécurité $F$ ne correspond pas au facteur de confiance $\Gamma$ et ceci, indépendamment de la méthode de calcul employée. Néanmoins, pour une même méthode de calcul (forme de frontière et calcul de $M_{\text {rés }}$ identiques), il existe une relation entre $\Gamma$ et $\mathrm{F}$ : pour la méthode de calcul présentée, $F$ est la valeur commune de $F_{\phi}$ et $F_{C}$ pour laquelle le facteur de confiance $\Gamma$ vaut 1 . Il est donc possible de déterminer $\mathrm{F}$ par approximations successives (en pratique, très peu d'itérations sont nécessaires). Inversement, toute méthode capable de calculer $F$ pourrait, moyennant quelques modifications, calculer $\Gamma$. Toutefois, les différences fondamentales qui subsistent au niveau du calcul (voir §3) font que les valeurs de $F$ ou de $\Gamma$ peuvent varier d'une méthode à l'autre.

A méthode de calcul égale, les notions de facteur de confiance et de coefficient de sécurité sont donc apparemment équivalentes. En fait, le facteur de confiance $\Gamma$ présente d'indéniables avantages sur le coefficient de sécurité $F$ :

- la notion de facteur de confiance permet de prendre en compte des coefficients de sécurité différents sur la cohésion et l'angle de frottement ;

- en pratique, lors du dimensionnement ou de la vérification d'un ouvrage, le problème n'est pas de connaître la valeur du coefficient de sécurité sur le sol F mais seulement de savoir s'il est supérieur ou inférieur à la valeur minimale $\mathrm{F}_{\min }$ imposée par la régle- 
mentation ou, à défaut, par le cahier des charges. Autrement dit, le problème n'est pas de déterminer $F$ mais bien de savoir si le facteur de confiance $\Gamma$ est supérieur ou inférieur à 1 lorsque $F_{\phi}=F_{C}=F_{\min }$; - dans le cas d'un ouvrage renforcé, la définition même du coefficient de sécurité $F$ peut être mise en défaut indépendament de la méthode de calcul employée: on peut montrer que le coefficient de sécurité $\mathrm{F}$ devient infini au-delà d'un certain niveau de renforcement. Le facteur de confiance $\Gamma$ ne présente pas cet inconvénient;

- dans le cas d'un sol hétérogène, le facteur de confiance permet de définir des coefficients de sécurité différents sur les capacités de résistance de chaque type de sol.

En somme, le coefficient de sécurité $F$, initialement défini pour les talus homogènes non renforcés, supporte mal d'être généralisé au cas des ouvrages renforcés. Le facteur de confiance $\Gamma$ est en revanche bien conçu pour tous les types d'ouvrages.

\section{MISE EN CEUVRE INFORMATIQUE}

La méthode présentée a été mise en œuvre dans un logiciel (STARS) conçu pour fonctionner en mode conversationnel sur micro-ordinateur. Nous présentons ci-après quelques aspects pratiques du logiciel (description des données, du calcul, des résultats et du support informatique).

\subsection{Les données}

La figure 6 présente les données acceptées par la version actuelle du logiciel. Probablement appelée à être complétée ultérieurement, cette liste de base est d'ores et déjà apte à décrire précisément un grand nombre de cas réels.

Le tableau 1 donne la définition, l'unité et, éventuellement, le domaine de variation des variables relatives à la géométrie, au chargement et aux capacités de résistance. On a choisi les conventions suivantes :

- les abscisses et les cotes sont relatives au repère Oxz représenté sur la figure 1 ;

- les angles sont comptés positivement dans le sens trigonométrique inverse ;

- les efforts sont comptés positivement vers le bas. Certains renseignements doivent être considérés à la lumière des hypothèses suivantes :

- les plans supérieur et inférieur du talus sont supposés semi-infinis. Ceci explique que leurs inclinaisons

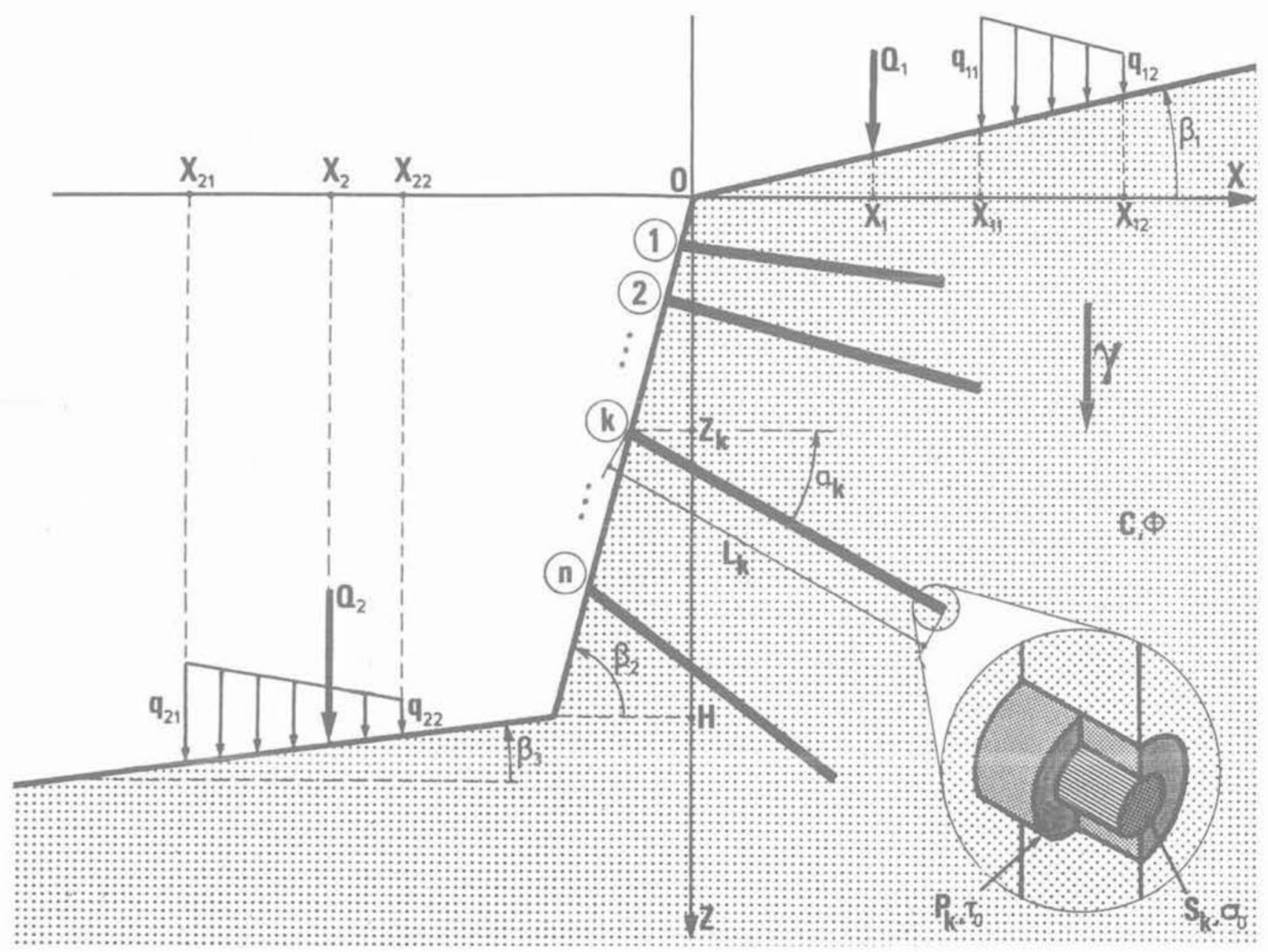

Fig. 6. - Ouvrage standard pour le programme STARS.

Fig. 6. - Standard structure for the programm STARS. 
Tableau 1. - Description des données définissant l'ouvrage

Table 1. - Specification of the data defining the structure

\begin{tabular}{|c|c|c|c|}
\hline Symbole & Définition & Unité & Restrictions \\
\hline $\mathrm{H}$ & Hauteur du talus & m & $H>0$ \\
\hline$\beta_{1}$ & Inclinaison du plan supérieur & Degré & $\beta_{1} \leq \phi$ et $\pi-\beta_{2}<\beta_{1}<\beta_{2}$ \\
\hline$\beta_{2}$ & Inclinaison du talus & Degré & $0<\beta_{2} \leq \pi / 2$ \\
\hline$\beta_{3}$ & Inclinaison du plan inférieur & Degré & $b_{3} \leq \phi$ et $\pi-\beta_{2}<\beta_{3}<\beta_{2}$ \\
\hline$\gamma$ & Poids volumique du sol (homogène) & $\mathrm{kN} / \mathrm{m}^{3}$ & $\gamma \geq 0$ \\
\hline$\phi$ & Angle de frottement interne (homogène) & Degré & $0 \leq \phi<\pi / 2$ \\
\hline C & Cohésion du sol (homogène) & $\mathrm{kPa}$ & $c \geq 0$ \\
\hline$Q_{i}$ & $i^{\mathrm{e}}$ surcharge linéique & $\mathrm{kN} / \mathrm{m}$ & \\
\hline$x_{i}$ & Abscisse du point d'application de $Q_{i}$ & m & $X_{i} \leq-H \operatorname{ctg} \beta_{2}$ ou $X_{i} \geq 0$ \\
\hline$q_{j 1}, q_{j 2}$ & Densités extrêmes de la $j^{\ominus}$ surcharge surfacique & $\mathrm{kPa}$ & \\
\hline$x_{i 1}, x_{j 2}$ & $\begin{array}{l}\text { Abscisses extrêmes de l'intervalle d'application } \\
\text { de } q_{i}\end{array}$ & m & $\begin{array}{l}X_{i 1} \neq X_{j 2} \text { et } \max \left(X_{j 1}, X_{i 2}\right) \leq-H \operatorname{ctg} \beta_{2} \\
\text { ou } \min \left(X_{i 1}, X_{j 2}\right) \geq 0\end{array}$ \\
\hline n & Nombre de lits d'inclusions & & $n \geq 0$ \\
\hline$\sigma_{0}$ & $\begin{array}{l}\text { Traction limite de I'acier constitutif } \\
\text { des inclusions }\end{array}$ & $\mathrm{kPa}$ & $\sigma_{0} \geq 0$ \\
\hline$\tau_{0}$ & Cisaillement limite à l'interface sol-inclusion & $\mathrm{kPa}$ & $\tau_{0} \geq 0$ \\
\hline$z_{k}$ & Cote du point d'émergence du $\mathrm{k}^{\mathrm{e}}$ lit d'inclusions & m & $0 \leq Z_{k} \leq H$ \\
\hline$\alpha_{k}$ & Inclinaison du $k^{e}$ lit d'inclusions & Degré & $-\beta_{2}<\alpha_{k}<\pi-\beta_{2}$ \\
\hline$L_{k}$ & Longueur du $\mathrm{k}^{\theta}$ lit $\mathrm{d}^{\prime}$ inclusions & m & $L_{k} \geq 0$ \\
\hline $\mathrm{S}_{\mathrm{k}}$ & Section équivalente du $\mathrm{k}^{\mathrm{e}}$ lit $\mathrm{d}^{\prime}$ inclusions & $\mathrm{cm}^{2} / \mathrm{m}$ & $s_{k} \geq 0$ \\
\hline$P_{k}$ & Périmètre équivalent du $\mathrm{k}^{\mathrm{e}}$ lit $\mathrm{d}^{\prime}$ inclusions & $\mathrm{cm} / \mathrm{m}$ & $P_{k} \geq 0$ \\
\hline
\end{tabular}

respectives ne puissent excéder l'angle de frottement interne du sol en valeur absolue;

- l'ouyrage est considéré sous l'hypothèse de déformation plane. C'est pour cette raison que certaines quantités sont données par mètre linéaire transversal $\left(Q_{i}, S_{k}, P_{k}\right)$;

- le sol est régi par un critère de Coulomb;

- les inclusions sont supposées ne résister qu'en traction, les résistances en compression, flexion et cisaillement étant a priori négligées. Toutefois, une option permet de prendre en compte la résistance en compression ;

- l'interface sol-inclusion est régie par un critère de Tresca ;

- chaque lit d'inclusions est caractérisé par une sec- tion équivalente $S_{k}$ exprimée en $\mathrm{cm}^{2} / \mathrm{m}$ et un périmètre extérieur équivalent $\mathrm{P}_{\mathrm{k}}$ exprimé en $\mathrm{cm} / \mathrm{m}$. Ils représentent respectivement la section d'acier d'une inclusion et son périmètre extérieur (éventuellement périmètre du coulis), tous deux rapportés à la distance séparant deux inclusions successives du même lit ; - en théorie, rien ne limite le nombre de surcharges et le nombre de lits d'inclusions. En pratique, le programme accepte jusqu'à 10 lits d'inclusions et 8 surcharges ( 4 linéiques et 4 surfaciques). Bien entendu, ce choix n'est pas irréversible mais il nous a semblé raisonnable compte tenu du volume de données à saisir et des temps de calcul observés.

Le tableau 2 récapitule les coefficients de sécurité partiels et leurs effets pondérateurs.

Tableau 2. - Coefficients de sécurité partiels

Table 2. - Partial safety coefficients

\begin{tabular}{|c|c|c|c|}
\hline Valeurs caractéristiques & Coefficients de sécurité & Valeurs pondérées & Restrictions \\
\hline$\phi$ & $F_{\phi}$ & $\operatorname{arctg}\left(\operatorname{tg} \phi / F_{\phi}\right)$ & $F_{\phi} \geq 1$ \\
\hline C & $\mathrm{F}_{\mathrm{C}}$ & $\mathrm{C} / \mathrm{F}_{\mathrm{C}}$ & $F_{C} \geq F_{\phi}$ \\
\hline$\sigma_{a}$ & $\mathrm{~F}_{\sigma_{0}}$ & $\sigma_{0} / F_{\sigma_{0}}$ & $\mathrm{~F}_{o_{0}} \geq 1$ \\
\hline$\tau_{0}$ & $F_{\tau_{0}}$ & $\tau_{0} / F_{\tau_{0}}$ & $F_{x_{0}} \geq 1$ \\
\hline$\gamma$ & $\mathrm{F}_{\gamma}$ & $\gamma \mathrm{F}_{\gamma}$ & $F_{\gamma} \geq 1$ \\
\hline $\mathrm{Q}_{i}$ & $\mathrm{~F}_{\mathrm{Q}_{i}}, \mathrm{~F}_{\mathrm{Q}_{i}}^{+}$ & {$\left[\mathrm{Q}_{i} \mathrm{~F}_{\mathrm{Q}_{i}} ; \mathrm{Q}_{\mathrm{i}} \mathrm{F}_{\mathrm{O}_{i}}^{+}\right]$} & $\mathrm{F}_{\mathrm{Q}_{\mathrm{i}}} \leq 1 \leq \mathrm{F}_{\mathrm{Q}_{\mathrm{i}}}^{+}$ \\
\hline$a_{j}$ & $\mathrm{~F}_{\mathrm{q}_{\mathrm{j}}}^{-}, \mathrm{F}_{\mathrm{q}_{\mathrm{j}}}^{+}$ & {$\left[\mathrm{q}_{j} \mathrm{~F}_{\mathrm{q}_{j}}^{-} ; \mathrm{q}_{j} \mathrm{~F}_{\mathrm{q}_{j}}^{+}\right]$} & $F_{q_{j}}^{-} \leq 1 \leq F_{q_{j}}^{+}$ \\
\hline
\end{tabular}




\subsection{Les résultats}

Les volumes limités par une spirale ou une droite constituent une famille dépendant de trois paramètres. Le rapport $\Gamma_{V}=M_{\text {rés }} / M_{\text {act }}$ est alors une fonction scalaire définie sur un sous-ensemble $E$ de $R^{3}$ (les paramètres doivent vérifier certaines conditions pour que le volume correspondant soit correctement défini). Par abus de langage, un triplet de E sera appelé volume. Le facteur de confiance $\Gamma$ de louvrage est donc le minimum sur $E$ de la fonction $\Gamma_{V}$ et l'élément de $\mathrm{E}$ où ce miminum est atteint est le volume critique. En général, la fonction $\Gamma_{V}$ n'est pas convexe sur E. Elle admet donc des minima locaux pour des volumes qui seront qualifiés de sous-critiques. Le volume critique est donc l'un des volumes souscritiques.

Pour un ouvrage renforcé par $\mathrm{n}$ lits d'inclusions, le logiciel détermine automatiquement $n+2$ volumes sous-critiques ainsi que les rapports $\Gamma_{V}$ associés :

- pour i variant de 1 à $n+1$, le volume souscritique numéro i est le moins stable parmi ceux dont la frontière commence sur le plan supérieur et débouche sur le parement juste au-dessus du ieme lit d'inclusions sans l'intercepter (le lit fictif numéro $n+1$ correspond au prolongement du plan inférieur). Ces volumes seront dits de type I (fig. 7);

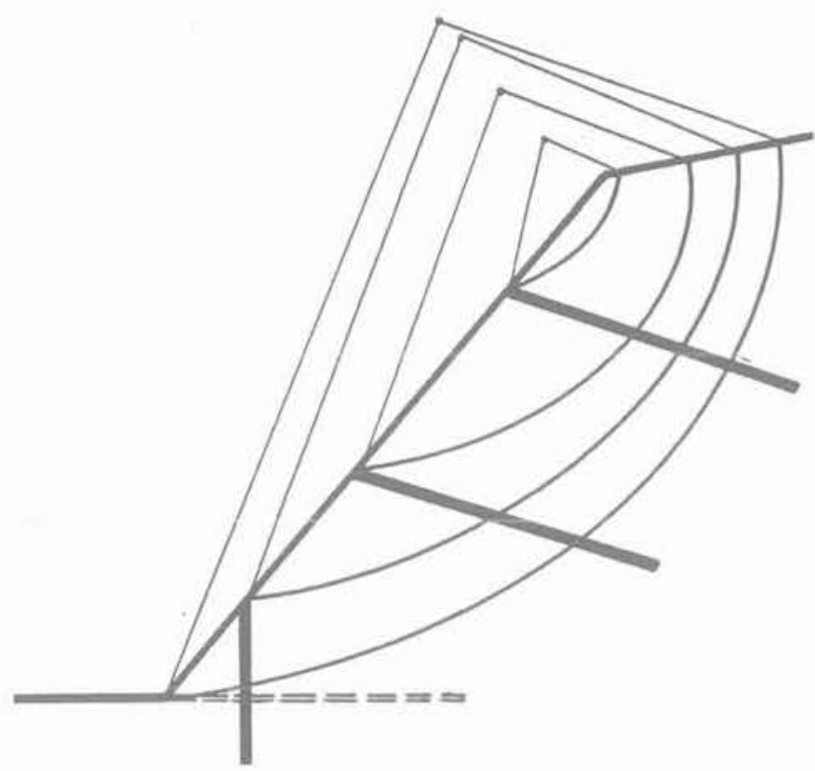

Fig. 7. - Volumes de type I. Fig. 7. - I-type volumes.

- le volume sous-critique numéro $n+2$ est le moins stable parmi ceux dont la frontière commence sur le plan supérieur et débouche sur le plan inférieur (ou au pied du talus en interceptant le lit fictif numéro $n+1)$. Ces volumes sont dits de type II (fig. 8).

Le facteur de confiance $\Gamma$ de l'ouvrage est alors égal au plus petit des $n+2$ rapports $\Gamma_{V}$ ainsi déterminés et le volume critique est celui qui réalise cette valeur minimale.

Bien entendu, dans le cadre d'une simple vérification de la stabilité d'un ouvrage, il suffit de connaître la

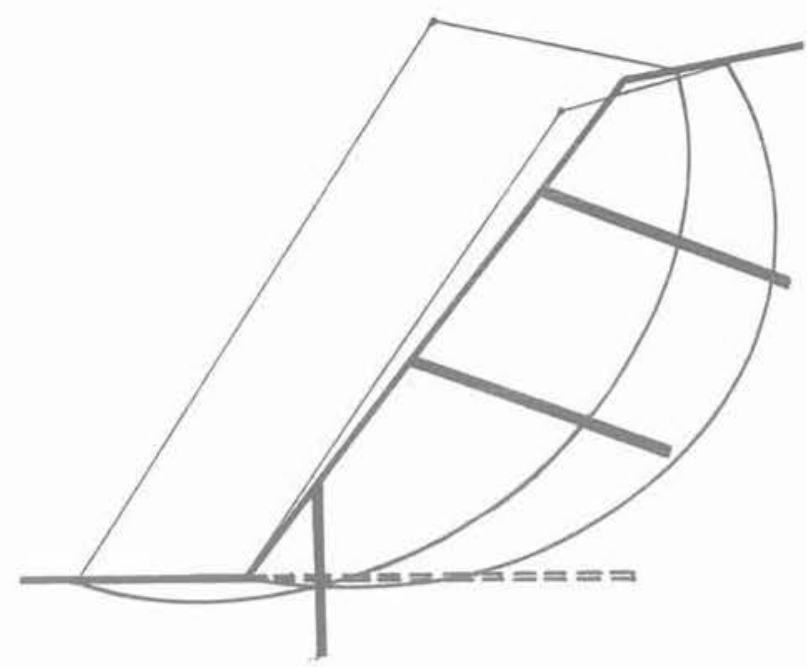

Fig. 8. - Volumes de type II.

Fig. 8. - II-type volumes.

valeur de son facteur de confiance $\Gamma$. En revanche, dès que l'on désire contrôler ou améliorer le rendement d'un schéma de renforcement, la connaissance des volumes sous-critiques et des rapports $\Gamma_{V}$ associés est fondamentale. Lors des exemples présentés dans le paragraphe 6 , nous verrons comment cette information permet de localiser les « points faibles » du renforcement.

\subsection{L'environnement informatique}

Le lecteur trouvera ici quelques indications qui lui permettront de se faire une idée concrète du mode de fonctionnement du logiciel conçu pour un microordinateur ( 640 Ko de RAM) sous-système d'exploitation MS-DOS, possédant un écran graphique et un coprocesseur scientifique. Le programme est écrit en FORTRAN (partie calcul) et en TURBOPASCAL (partie graphique).

La saisie des données simplifie la tâche de l'utilisateur. Tout se passe comme si ce dernier devait remplir une fiche de renseignement sur le problème qu'il désire étudier. La fiche apparaît sur l'écran, accompagnée d'un schéma explicatif. L'utilisateur peut remplir la fiche dans n'importe quel ordre et peut la modifier à volonté. Etant donné le nombre important de paramètres à définir, la fiche de renseignement est divisée en cinq rubriques qui apparaissent successivement à l'écran :

- louvrage et le sol $\left(\mathrm{H}, \beta_{1}, \beta_{2}, \beta_{3}, \gamma, \phi\right.$ et $\left.\mathrm{C}\right)$;

- les charges concentrées $\left(Q_{1}\right.$ et $\left.X_{1}\right)$;

- les charges réparties $\left(q_{j 1}, q_{j 2}, X_{j 1}\right.$ et $\left.X_{j 2}\right)$;

- le renforcement ( $\mathrm{n}, \sigma_{\mathrm{o}}, \tau_{\mathrm{o}}, \mathrm{Z}_{\mathrm{k}}, \alpha_{\mathrm{k}}, \mathrm{L}_{\mathrm{k}}, \mathrm{S}_{\mathrm{k}}$ et $\mathrm{P}_{\mathrm{k}}$ ) :

- les coefficients de sécurité partiels sur les résistances $\left(\mathrm{F} \phi, \mathrm{F}_{\mathrm{C}}, \mathrm{F}_{\sigma_{\mathrm{o}}}\right.$ et $\left.\mathrm{F}_{\tau_{\mathrm{o}}}\right)$ et sur le chargement $\left(\mathrm{F}_{\gamma}\right.$, $\mathrm{F}_{\mathrm{Q}_{i}}{ }^{-}, \mathrm{F}_{\mathrm{Q}_{\mathrm{i}}}{ }^{+}, \mathrm{F}_{\mathrm{q}_{j}}{ }^{-}$et $\mathrm{F}_{\mathrm{q}_{j}}{ }^{+}$). 
Lorsqu'une rubrique est complète, le programme vérifie si elle est cohérente avec les restrictions du tableau 1 ou 2. Lorsque la valeur d'un paramètre ne satisfait pas les conditions qui lui sont prescrites, le programme envoie un message qui peut être de deux types:

- un message de type "erreur » signifie que le calcul du facteur de confiance $\Gamma$ est impossible. Dans ce cas, l'utilisateur est contraint de modifier la donnée fautive ;

- un message de type " avertissement " signifie que le facteur de confiance $\Gamma$ peut être calculé à condition que la donnée concernée ne soit pas prise en compte. Ainsi, à chaque fois qu'une surcharge ou un lit d'inclusions n'est pas correctement défini, le calcul sera effectué sans en tenir compte. Il n'est donc pas nécessaire de préciser le nombre de surcharges. Nous avons néanmoins conservé le nombre de lits d'inclusions pour pouvoir étudier très commodément les phases de construction (voir l'exemple 6.2.).

Lorsqu'il n'y a plus de messages d'erreur dans aucune des cinq rubriques, l'utilisateur a le choix entre deux options non exclusives:

- soit il demande une visualisation de l'ouvrage qui lui permet de vérifier rapidement que le jeu de données correspond bien à l'ouvrage qu'il désire étudier ;

- soit il demande directement le calcul du facteur de confiance $\Gamma$. Les volumes sous-critiques de type I et II sont déterminés séparément et sur la demande de l'utilisateur. Il arrive en effet fréquemment que l'un ou l'autre type de volumes ne soit pas pertinent pour l'ouvrage étudié.

Le temps de calcul est étroitement lié à la capacité de l'ordinateur. Sur un micro-ordinateur de type AT, il varie de quelques secondes à quelques minutes suivant que la sécurité est ou non prise en compte et suivant les nombres de lits d'inclusions et de surcharges.

A l'issue d'un calcul complet, le programme fournit donc les $n+2$ rapports $\Gamma_{y}$ correspondant aux $n+2$ volumes sous-critiques définis au paragraphe 5.2 ., le plus petit d'entre eux étant le facteur de confiance $\Gamma$ de l'ouvrage. L'utilisateur peut alors demander une représentation complète de louvrage et des volumes sous-critiques qui viennent d'être déterminés, le volume critique étant repréré d'une couleur différente.

Les trois étapes du programme (saisie des données, calcul, visualisation) sont, dans une certaine mesure, indépendantes. L'utilisateur peut, à tout moment, modifier les données, calculer le facteur de confiance ou encore, visualiser l'ouvrage et, éventuellement, les volumes sous-critiques déterminés à l'étape précédente. Le mode d'utilisation choisi dépendra du but recherché (vérification, dimensionnement, optimisation) comme nous allons le voir au cours des exemples du paragraphe suivant.

\section{EXEMPLES D’UTILISATION}

Les deux exemples retenus l'ont été pour des raisons bien différentes: le premier a surtout une valeur pédagogique dans la mesure où il permet de faire l'apprentissage du logiciel ; le second est une simulation réaliste d'un cas tel qu'il pourrait se présenter à un ingénieur.

\subsection{Position et longueur optimales d'un lit d'inclusion}

Le but de ce premier exemple est de montrer comment utiliser les volumes sous-critiques et les rapports $\Gamma_{V}$ associés de manière à optimiser un schéma de renforcement. Par souci de clarté, nous avons délibérément choisi d'étudier un ouvrage très simple : il s'agit d'un talus vertical pesant reposant sur un substratum rigide et susceptible d'être renforcé par un seul lit d'inclusions horizontales. De plus, certaines caractéristiques sont imposées a priori (fig. 9)

Par ailleurs, tous les coefficients de sécurité partiels sont égaux à l'unité. L'optimisation du schéma de renforcement ne porte donc que sur deux paramètres à savoir la profondeur $\mathrm{Z}$ et la longueur $\mathrm{L}$ des inclusions. Plus précisément, nous nous proposons de déterminer successivement :

- la profondeur $Z_{\text {opt à laquelle doivent étre placées }}$ les inclusions supposées infiniment longues pour que le facteur de confiance $\Gamma$ de l'ouvrage soit maximal ; - à cette profondeur $Z_{\text {opt }}$, la longueur $L_{\text {opt }}$ des inclusions au-dessous de laquelle le facteur de confiance $\Gamma_{\text {opt }}$ obtenu à l'étape précédente n'est plus atteint.

$$
\begin{array}{ll}
\gamma=20 \mathrm{kN} / \mathrm{m}^{3} & \sigma_{0}=200 \\
\Phi=30^{\circ} & S=5 \mathrm{~cm} \\
\mathrm{C}=10 \mathrm{kPa} & \mathbf{P}=10 \mathrm{~cm} \\
T_{0}=+\infty \text { (adhérence parfaite) }
\end{array}
$$

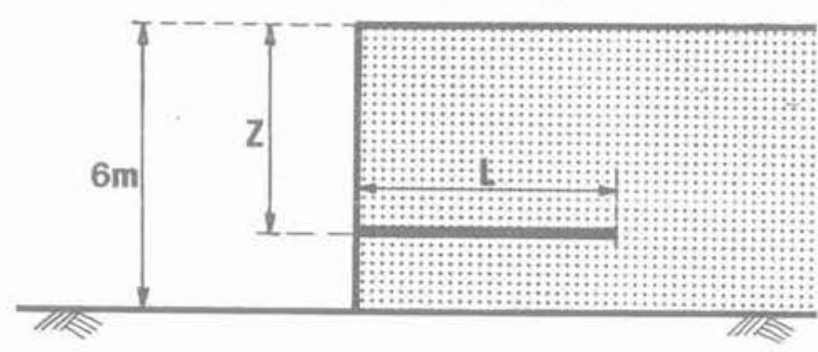

Fig. 9. - Détermination des positions et longueur optimales d'un lit d'inclusions.

Fig. 9. - Determination of the optimal position and length of a layer of inclusions.

Huit passages informatiques effectués pour différentes valeurs du couple $(\mathrm{Z}, \mathrm{L})$ ont été sélectionnés pour leur caractère représentatif. Sur la figure 10 sont reproduits les résultats tels qu'ils apparaissent à l'utilisateur : sur un schéma de louvrage sont représentés le(s) volume(s) sous-critique(s) de type I (la présence du substratum rigide élimine a priori le volume souscritique de type II). Le volume critique correspond à la frontière continue, les volumes sous-critiques éventuels étant représentés en pointillés. Le rapport $\Gamma_{V}$ de chaque volume est indiqué en regard du point du parement où débouche sa frontière et le facteur de confiance $\Gamma$ est rappelé en haut à gauche. Enfin, le temps de calcul sur un micro-ordinateur compatible 
de type AT est indiqué en secondes dans un encadré en haut à droite.

Le premier passage (fig. 10.a.) concerne le talus non renforcé. Le seul volume sous-critique déterminé est aussi le volume critique et sa frontière passe par le pied du talus. On vérifie que la valeur obtenue pour le facteur de confiance $(\Gamma=0,56)$ coïncide avec celle calculée à partir d'abaques fondées sur la théorie des charges limites (voir par exemple CHEN, 1975).

Pour les deux passages suivants (fig. 10.b. et 10.c.), les inclusions, supposées infiniment longues, ont été placées à deux profondeurs différentes $(Z=2 \mathrm{~m}$, puis $Z=4 \mathrm{~m}$ ). Dans les deux cas, on obtient deux volumes sous-critiques de type I : la frontière de l'un débouche juste au-dessus du lit d'inclusions alors que celle de l'autre passe par le pied du talus. Suivant la profondeur Z du lit d'inclusions, c'est l'un ou l'autre de ces deux volumes qui devient critique mais, dans les deux cas, le facteur de confiance $\Gamma$ est amélioré par rapport à la configuration 10.a. : de 0,56 , il passe à 1,18 lorsque $Z=2 \mathrm{~m}$ et à 0,84 lorsque $Z=4 \mathrm{~m}$. Le meilleur facteur de confiance $\Gamma_{\mathrm{opt}}$, c'est-à-dire le plus élevé, sera donc obtenu pour la profondeur $Z_{\text {opt }}$ telle que les rapports $\Gamma_{V}$ des deux volumes souscritiques soient égaux (fig. 10.d.). Cette profondeur optimale $Z_{\text {opt }}$ peut être déterminée avec toute la précision voulue par approximations successives (dans l'exemple traité, $Z_{\text {opt }}=2,59 \mathrm{~m}$ au centimètre près!). Pour cette valeur de $Z$, le facteur de confiance atteint sa valeur maximale $\Gamma_{\text {opt }}=1,29$.

Les derniers passages (fig. 10.e, à 10.h.) concernent l'optimisation de la longueur des inclusions. La profondeur $\mathrm{Z}$ étant alors fixée à sa valeur optimale $Z_{\text {opt }}$ $=2,59 \mathrm{~m}$, l'objectif est de déterminer la longueur minimale $L_{\text {opt }}$ que doivent avoir les inclusions pour que le facteur de confiance $\Gamma_{\text {opt }}$ soit atteint. Le premier passage, effectué avec $\mathrm{L}=5 \mathrm{~m}$ (fig. 10.e.), conduit au même résultat que le passage avec une longueur infinie (les volumes et les rapports $\Gamma_{V}$ associés sont identiques). Il permet donc d'affirmer que $\mathrm{L}_{\text {opt }}$ est inférieure à $5 \mathrm{~m}$. En revanche, le second passage, effectué avec $L=3 \mathrm{~m}$ (fig. 10.f.), indique une chute du facteur de confiance de 1,29 à 0,64 , ce qui permet d'affirmer que $\mathrm{L}_{\mathrm{opt}}$ est supérieure à 3 $\mathrm{m}$. Par rapport au passage précedent, seul le volume sous-critique dont la frontière passe par le pied du talus a été modifié. Il redevient le seul volume critique avec cette particularité que les inclusions ne sont plus sollicitées. Ceci ne signifie pas que le renforcement soit inefficace : la comparaison des figures 10.a. et 10.f. montre que le facteur de confiance est amélioré par les inclusions (il passe de 0,56 à 0,64 ), bien que ces dernières ne contribuent pas à la stabilité du volume critique. En fait, elles y contribuent indirectement en

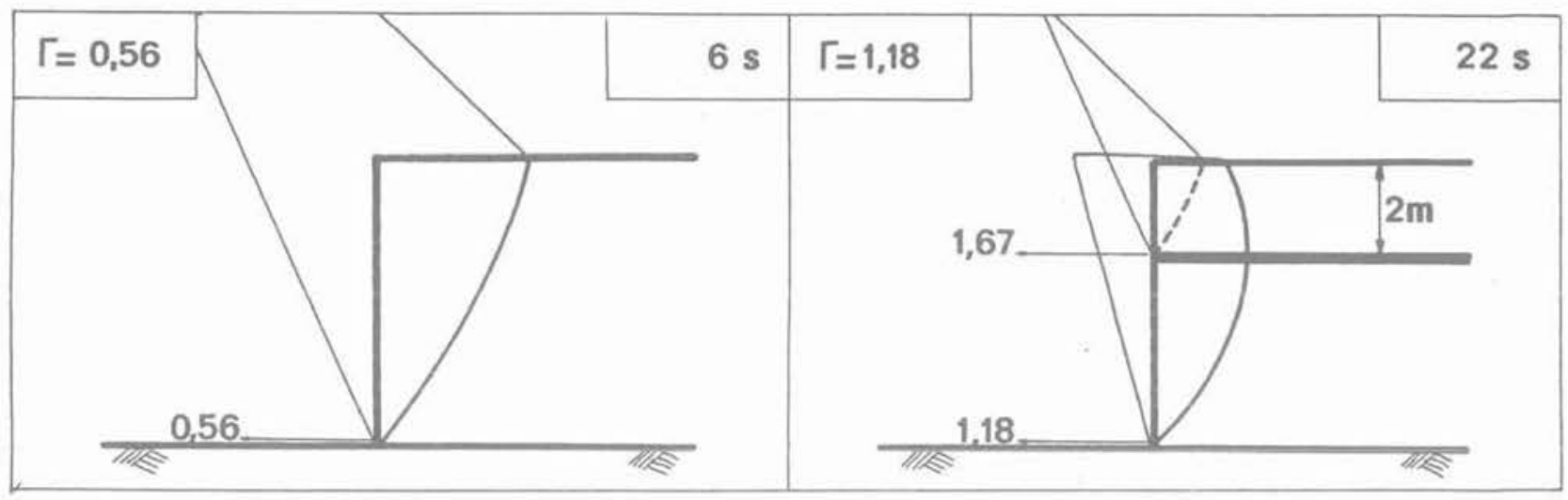

Fig. 10.a. - Ouvrage non renforcé.

Fig. 10.a. - Unreiforced structure.
Fig, 10.b. - Les inclusions sont trop hautes.

Fig. 10.b. - The inclusions are too high.

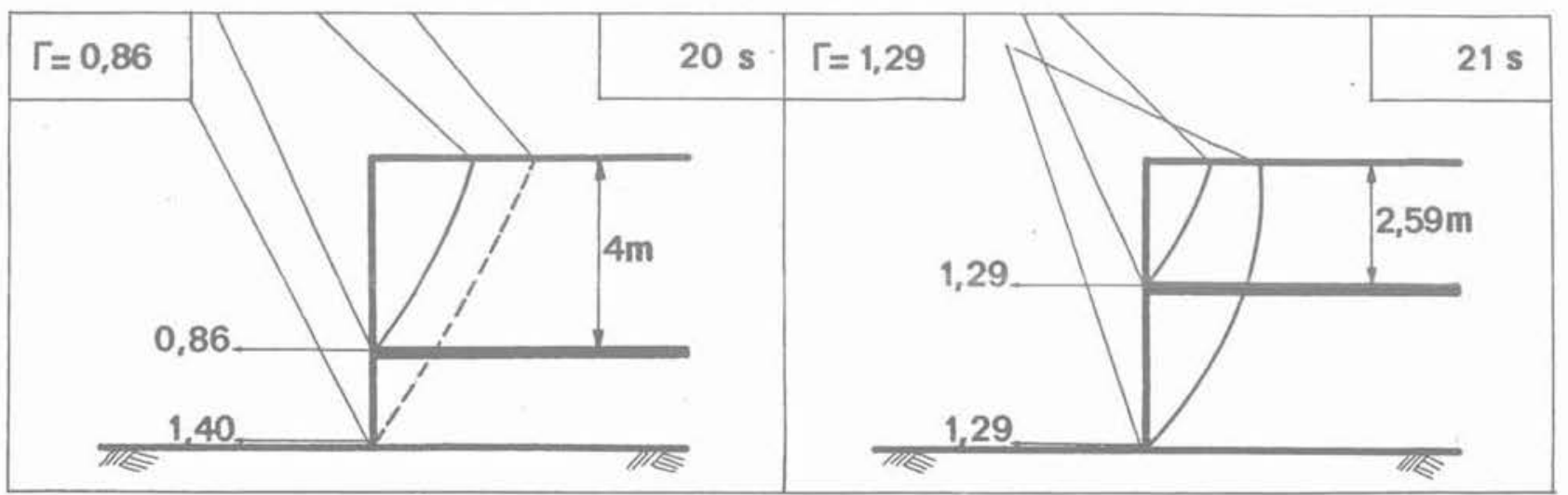

Fig. 10.c. - Les inclusions sont trop basses. Fig. 10.c. - The inclusions are too low.
Fig. 10.d. - La position des inclusions est optimale. Fig. 10.d. - The position of the inclusions is optimal. 


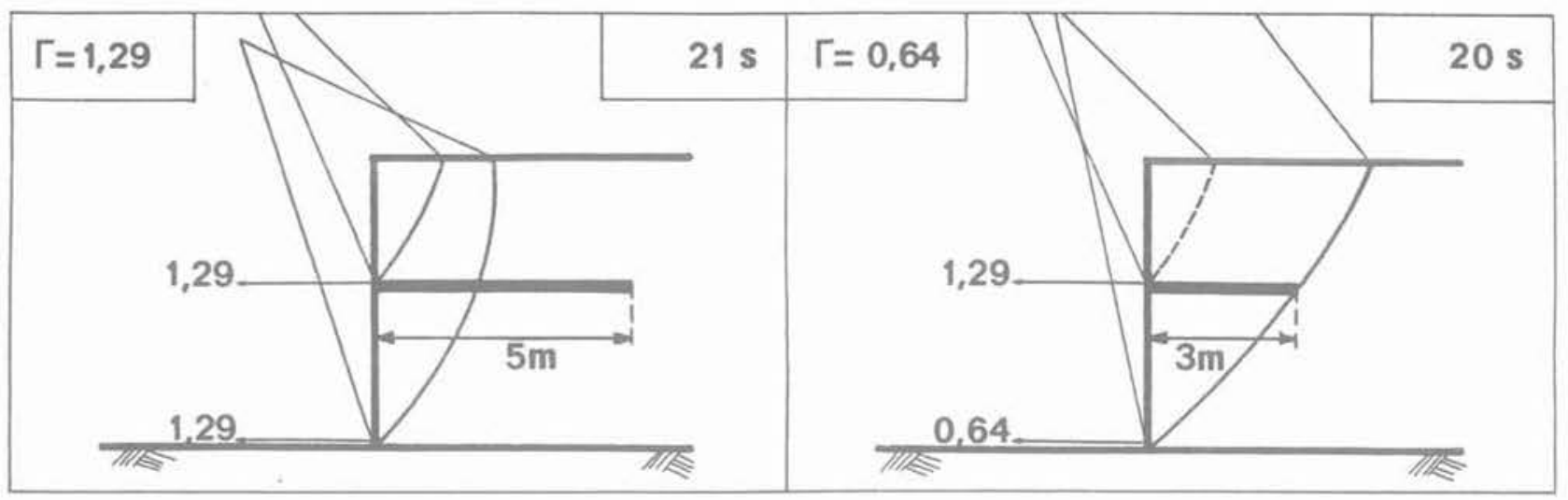

Fig. 10.e. - Les inclusions sont trop longues. Fig. 10.e. - The inclusions are too long.

Fig. 10.f. - Les inclusions sont trop courtes.

Fig. 10.t - The inclusions are too short.

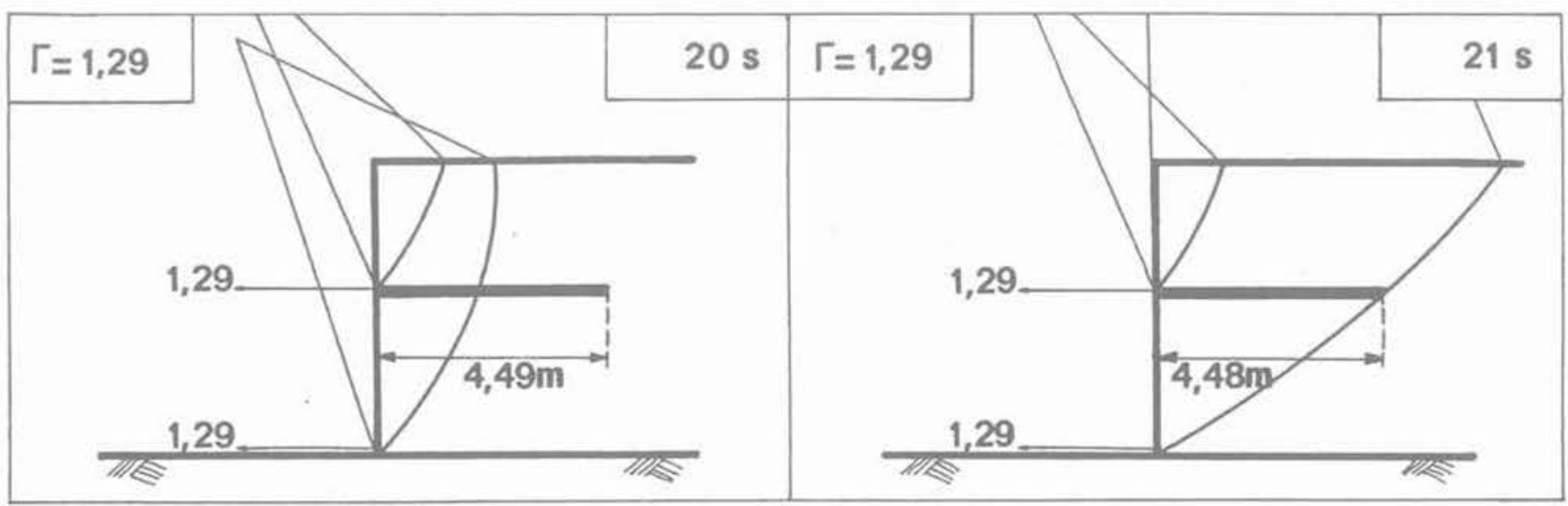

Fig. 10.g. - Les inclusions sont un peu trop longues. Fig. 10.9 . - The inclusions are slightly too long.

Fig. 10.h. - Les inclusions sont un peu trop courtes. Fig. 10.h. - The inclusions are slightly too short.

Fig. 10. - Résultats des passages informatiques.

Fig. 10. - Results of the computation.

déviant la frontière du volume critique relatif au talus non renforcé. Finalement, la longueur optimale $\mathrm{L}_{\text {opt }}$ pourra être obtenue par approximations successives. Théoriquement, elle est caractérisée par la coexistence de trois volumes critiques à savoir les deux de la figure $10 . \mathrm{d}$. et un troisième dont la frontière passe par le pied du talus sans couper les inclusions. En pratique, le programme ne peut pas fournir ces trois volumes simultanément mais la longueur L est une évaluation par excès de $\mathrm{L}_{\mathrm{opt}}$ à $\Delta \mathrm{L}$ près dès que les résultats obtenus pour $\mathrm{L}$ et $\mathrm{L}-\Delta \mathrm{L}$ sont respectivement similaires à ceux des passages 10.e. et 10.f. On a ainsi obtenu $\mathrm{L}_{\text {opt }}=4,49 \mathrm{~m}$ (au centimètre près comme en attestent les passages effectués pour $\mathrm{L}=$ $4,49 \mathrm{~m}$ (fig. 10.g.) et $\mathrm{L}=4,48 \mathrm{~m}$ (fig, 10.h.) !).

Cet exemple élémentaire montre comment une bonne interprétation des résultats du programme permet de guider l'utilisateur lors de la conception et de l'optimisation d'un schéma de renforcement. Nous nous sommes limités à deux aspects particuliers du dimensionnement mais le même exemple pourrait faire l'objet d'une étude beaucoup plus générale. En effet, l'analyse dimensionnelle du problème révèle que le facteur de confiance $\Gamma$ de louvrage s'écrit sous la forme :

$\Gamma=\frac{C}{\gamma H} H\left[\beta_{1}, \beta_{2}, \beta_{3}, \phi, \frac{Z}{H}, \alpha, \frac{L}{H}, \frac{\sigma_{o} S}{C H}, \frac{\tau_{0} P}{C}\right]$

où $\mathrm{K}$ est une fonction sans dimension. Cette forme nous invite à faire une étude paramétrique du facteur de confiance $\Gamma$ en fonction des arguments adimensionnels de $\mathrm{K}$. On pourrait ensuite envisager de maximiser $\Gamma$ par rapport à ces mêmes arguments (nous lavons fait par rapport à $\mathrm{Z} / \mathrm{H}$ et $\mathrm{L} / \mathrm{H}$ successivement, pour des valeurs particulières des autres paramètres) sans oublier que le choix mais aussi lordre des paramètres d'optimisation à des résultats différents.

Une étude aussi complète n'est matériellement plus possible dès qu'il y a plus d'un lit d'inclusions. Le nombre de paramètres devient très vite prohibitif. II revient à chaque utilisateur de choisir sa propre stratégie de dimensionnement en fonction du cas traité, 
des contraintes techniques et économiques et aussi en fonction de sa propre expérience. Nous allons voir, sur l'exemple suivant, comment concilier contraintes de réalisation et optimisation.

\subsection{Dimensionnement et optimisation d'une fouille clouée}

En l'absence d'un jeu complet de données relatives à un ouvrage existant, l'exemple traité a été composé à partir des caractéristiques de plusieurs cas réels. Les caractéristiques connues de l'ouvrage à réaliser sont indiquées sur la figure 11.

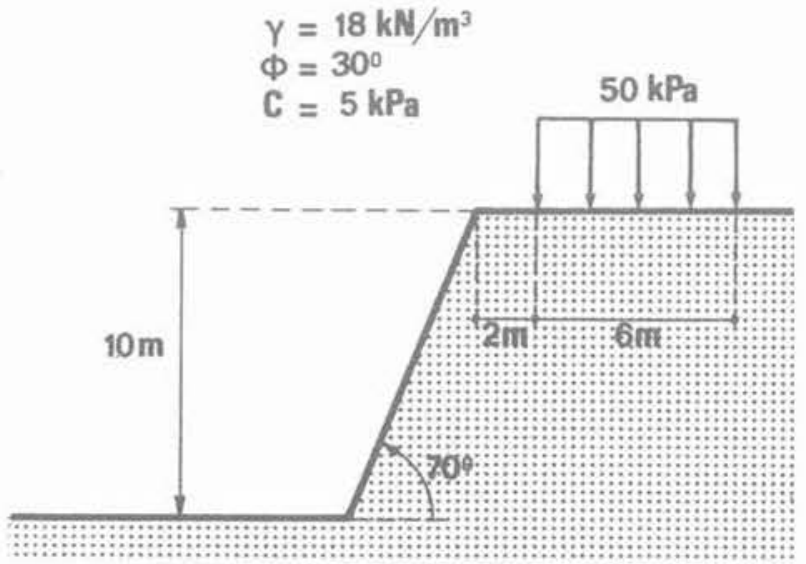

Fig. 11. - Dimensionnement et optimisation d'un talus.

Fig. 11. - Design and optimization of an embankment.

On dispose, pour le renforcer, de clous $\varnothing 16$ ( $\mathrm{S}=$ $\left.2 \mathrm{~cm}^{2}\right)$ et $\varnothing 32\left(\mathrm{~S}=8 \mathrm{~cm}^{2}\right)$, en acier de résistance $250000 \mathrm{kPa}$, qui seront mis en place dans des forages $\varnothing 64(\mathrm{P}=20 \mathrm{~cm})$, inclinés à 10 degrés sous l'horizontale puis injectés. Un essai d'arrachement a permis d'évaluer la résistance de l'interface ciment-sol $\left(\tau_{0}=100 \mathrm{kPa}\right)$. L'espacement horizontal entre deux clous est fixé à un mètre et la profondeur de forage est limitée à $12 \mathrm{~m}$.

Les coefficients de sécurité partiels exigés pour l'ouvrage définitif sont les suivants :

$\mathrm{F}_{\phi}=1,20, \mathrm{~F}_{\mathrm{C}}=1,50$;

$\mathrm{F}_{\mathrm{o}_{0}}=1,50, \mathrm{~F}_{\tau_{0}}=1,50$

$\mathrm{F}_{\gamma}=1,05, \mathrm{~F}_{\mathrm{q}}{ }^{-}=1,00, \mathrm{~F}_{\mathrm{q}}^{+}=1,10$;

Durant la réalisation de l'ouvrage, la surcharge q est maintenue mais les coefficients de sécurité partiels exigés sont moins pénalisants :

$\mathrm{F}_{\phi}=1,10, \mathrm{~F}_{\mathrm{C}}=1,30$;

$\mathrm{F}_{\sigma_{\mathrm{o}}}=1,30, \mathrm{~F}_{\tau_{\mathrm{o}}}=1,30$

$\mathrm{F}_{\gamma}=1,00, \mathrm{~F}_{\mathrm{q}}{ }^{-}=1,00, \mathrm{~F}_{\mathrm{q}}{ }^{+}=1,05$

S'il y a $\mathrm{n}$ lits de clous, la construction sera réalisée en $(n+1)$ terrassements successifs: avant de placer le $\mathrm{k}^{\text {eme }}$ lit de clous à la profondeur $Z_{\mathrm{k}}$, le terrain sera déblayé jusqu'à la profondeur $Z_{k}+0,50$ mètre.
Enfin une étude de coût a montré que les facteurs économiquement pénalisants sont, dans lordre d'importance décroissante :

- le nombre de forages par mètre linéaire transversal ;

- la longueur totale de forage:

- la quantité d'acier.

Le but de l'étude est donc de déterminer le schéma de renforcement le plus économique tel que le facteur de confiance de l'ouvrage soit au moins égal à l'unité, aussi bien en phase définitive qu'en phase provisoire. En pratique, ce type de problème sera traité en deux temps: le schéma de renforcement est tout d'abord optimisé vis-à-vis de la phase définitive puis vérifié en phase provisoire, cette dernière étant en général moins pénalisante.

Avant toute chose, il est toujours utile d'effectuer un premier calcul pour l'ouvrage non renforcé, ne seraitce que pour confirmer la nécessité d'un renforcement : on obtient $\Gamma_{0}=0,13$ pour le volume critique de type 1 représenté sur la figure 12 , ce qui est très insuffisant. Etant donnée la pente du parement, il n'est pas nécessaire de rechercher le volume souscritique de type II, d'autant que le temps de calcul correspondant est assez important. Cette recherche ne sera effectuée qu'une fois, à titre de vérification, sur l'ouvrage optimal c'est-à-dire en fin de dimensionnement.

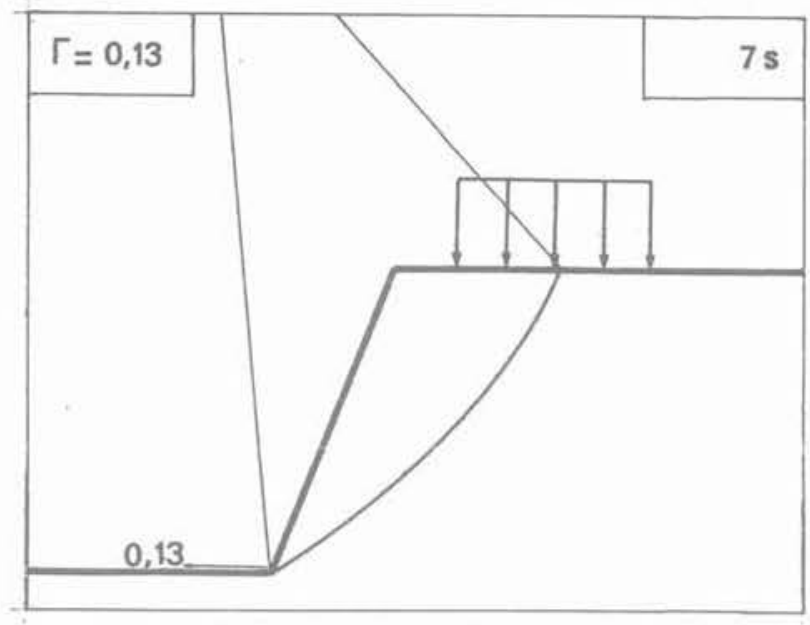

Fig. 12. - Ouvrage non renforce.

Fig. 12. - Unreinforced structure.

Pour estimer grossièrement la quantité d'acier nécessaire, on peut utiliser la règle semi-empirique suivante: pour que le facteur de confiance $\Gamma_{0}$ d'un ouvrage non renforcé passe à une valeur $\Gamma$ après renforcement, la section d'acier nécessaire par mètre linéaire transversal s est approximativement donnée par:

$s=2 \mathrm{CH}\left(\Gamma / \Gamma_{0}-1\right) / \sigma_{0}$, si $\Gamma_{0} \neq 0$,

ou

$\mathrm{s}=2 \mathrm{H} \Gamma / \Gamma_{\text {ref }}$, si $\Gamma_{0}=0, \Gamma_{\text {ref étant le facteur de }}$ confiance obtenu pour une cohésion unitaire. 
Cette estimation découle de deux approximations successives :

- tout d'abord, la section d'acier s est supposée uniformément répartie sur toute la hauteur $\mathrm{H}$ (processus d'homogénéisation). Le critère de résistance homogène correspondant est alors anisotrope ([SIAD, 1987], [DE BUHAN et al., 1987]) ;

- ensuite, ce critère est majoré par le critère de Coulomb d'angle $\phi$ et de cohésion $\mathrm{C}+\sigma_{\mathrm{o}} \mathrm{s} / 2 \mathrm{H}$.

Par ailleurs, l'analyse dimensionnelle permet de montrer que le facteur de confiance d'un ouvrage constitué d'un sol homogène de Coulomb est proportionnel à la cohésion, tout autre paramètre restant constant $\left(\gamma, H, \phi, Q_{i}, q_{i}\right.$, etc.). On obtient donc :

$\Gamma_{0}=C \Gamma_{\text {ref }}$ pour l'ouvrage non renforcé, et

$\Gamma \approx\left(\mathrm{C}+\sigma_{o} \mathrm{~s} / 2 \mathrm{H}\right) \Gamma_{\text {ref }}$ pour l'ouvrage renforcé,

d'où l'on tire facilement l'estimation de s qui peut être par excès ou par défaut car du processus d'homogénéisation résulte en général une diminution des capacités de résistance.

Dans le cas de l'ouvrage étudié, il faut utiliser les capacités de résistance réduites $\sigma_{0} / \mathrm{F}_{\sigma_{0}}$ et $\mathrm{C} / \mathrm{F}_{\mathrm{C}}$, si bien que :

$s \simeq 2 \times 5 \times(10 / 1,50)$

$\times(1 / 0,13-1) /(250000 / 1,50)=0,0027 \mathrm{~m}^{2} / \mathrm{m}$

que l'on répartit en quatre lits d'inclusions, trois de clous $\varnothing 32$ et un de clous $\varnothing 16$ (on choisit en priorité les clous les plus gros pour limiter le nombre de forages). Les inclusions les plus hautes étant les moins sollicitées, le lit de clous $\varnothing 16$ est placé à deux mètres de profondeur et ceux de clous $\varnothing 32$ à quatre, six et huit mètres. Enfin, les longueurs des clous sont toutes choisies maximales $(\mathrm{L}=12 \mathrm{~m}$ ) afin d'obtenir le meilleur ancrage possible. Pour cette configuration, le facteur de confiance est égal à 0,85 ce qui semble indiquer que le renforcement est insuffisant (fig. 13).

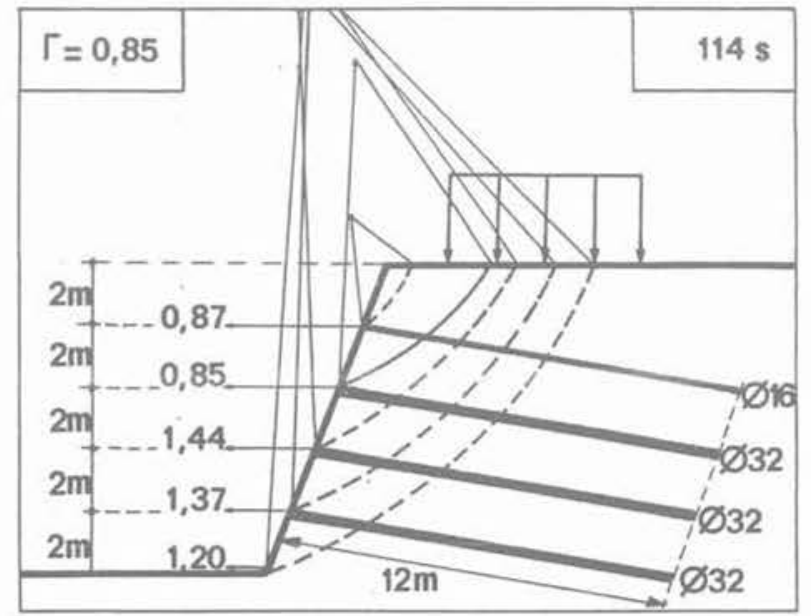

Fig. 13. - Mauvaise répartition des lits d'inclusions. Fig. 13. - Bad distribution of the inclusion layers.
En réalité, les cinq volumes sous-critiques obtenus et les rapports $\Gamma_{V}$ associés montrent que les lits d'inclusions sont mal répartis (le haut de talus est plus faible que le bas). Le facteur de confiance peut alors être amélioré en ne jouant que sur la position des lits et ceci, tant que l'un des volumes sous-critiques conduit à un rapport $\Gamma_{V}$ supérieur au facteur de confiance de l'ouvrage. La meilleure disposition des quatre lits, obtenue par approximations successives, conduit à un facteur de confiance égal à 1,20 (fig. 14) et l'égalité de tous les rapports $\Gamma_{V}$ prouve que la répartition du renforcement est optimale.

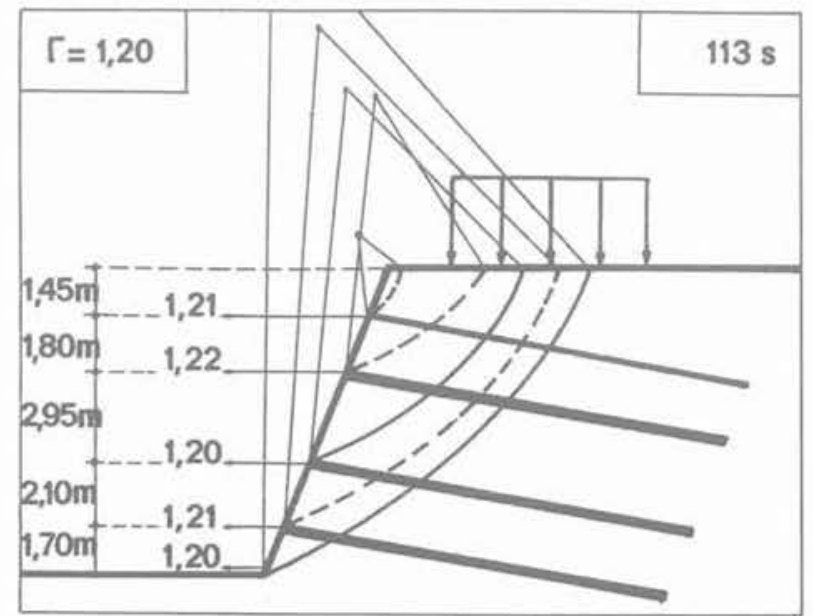

Fig. 14. - Répartition optimale des lits d'inclusions. Fig. 14. - Optimal distribution of the inclusion layers.

Cependant, l'ouvrage est alors surdimensionné puisque son facteur de confiance est largement supérieur à l'unité. Il est donc possible de réduire son coût en diminuant les sections d'acier ou les longueurs de forage et en modifiant à nouveau la disposition, Après quelques tatonnements guidés par les impératifs économiques (nombre minimal de forages), la configuration de la figure 15 semble être la meilleure dans la mesure où seulement trois lits de clous sont nécessaires.

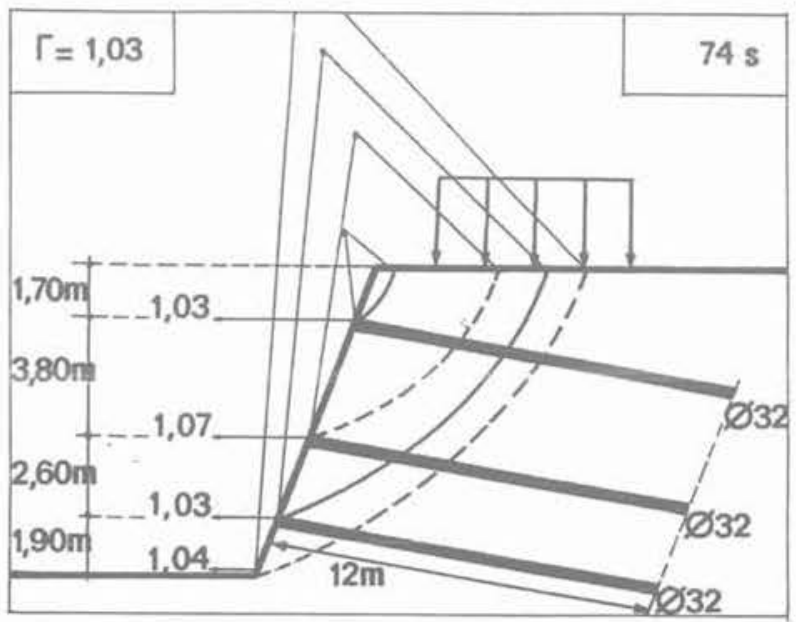

Fig. 15. - Schéma de renforcement optimal. Fig. 15. - Optimal reinforcement scheme. 
Le facteur de confiance $\Gamma$ est alors légèrement supérieur à l'unité $(1,03)$ et le renforcement est réparti au mieux (les rapports $\Gamma_{V}$ sont sensiblement égaux). On peut par ailleurs vérifier que l'équilibre du volume sous-critique de type II est bien assuré : il possède un rapport $\Gamma_{V}$ égal à 1,35 (fig. 16). Ce dernier calcul est sensiblement plus long et n'est pas pertinent pour le cas traité puisque le rapport $\Gamma_{V}$ excède largement le facteur de confiance de l'ouvrage. Il peut en revanche s'avérer déterminant dans certains cas (angle de frottement et inclinaison de talus très faibles).

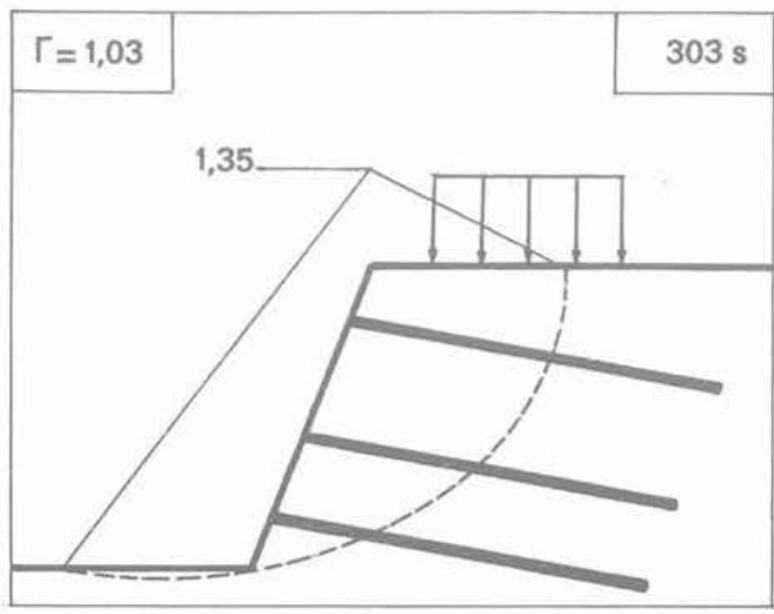

Fig. 16. - Volume sous-critique de type II. Fig. 16. - Sub-critical volume of type II.

C'est donc la solution de la figure 15 qui est retenue. Il reste maintenant à vérifier que les trois phases de terrassement sont stables, compte tenu de la sécurité exigée pendant la construction.

En pratique, le contrôle de la $\mathrm{i}^{\text {eme }}$ phase peut être effectué en relançant le programme après avoir modifié de manière adéquate les données relatives à l'ouvrage définitif :

- les coefficients de sécurité partiels doivent être actualisés à leurs valeurs en phase de construction ;

- la hauteur $\mathrm{H}$ doit être remplacée par la hauteur de sol déblayée à la phase considérée $\left(Z_{1}+0,50 \mathrm{~m}\right.$ dans le cas traité) :

- le nombre de lits d'inclusions à prendre en compte doit être explicitement fixé à i-1. En effet, bien que le $i^{\text {eme }}$ lit soit correctement défini $(0 \leq Z \leq H$ $\left.=Z_{i}+0,50 \mathrm{~m}\right)$, il ne doit pas être pris en compte dans le calcul puisqu'il n'est pas encore mis en place.

Lors de la vérification des données, le programme détecte le fait que i lits d'inclusions sont correctement définis alors que seulement (i-1) sont demandés. Il choisit alors automatiquement de ne prendre en compte que les (i-1) lits les moins profonds. De plus, le seul volume sous-critique de type I recherché est celui dont la frontière passe par la base du talus en terrassement. En effet, l'équilibre des autres volumes de type I est forcément assuré puisqu'il l'était déjà en phase définitive, c'est-à-dire pour des coefficients de sécurité partiels plus pénalisants.
Pour l'ouvrage retenu, les trois phases de construction et les facteurs de confiance correspondants sont représentés sur la figure 17. La phase la plus critique est la première $(\Gamma=1,03)$ alors que les deux suivantes sont très largement stables $(\Gamma=1,20$ et $1,24)$. L'ouvrage respecte donc la sécurité exigée en phase provisoire. Si tel n'avait pas été le cas, il eût été très facile de modifier à nouveau le renforcement, puis de vérifier l'ouvrage en phase provisoire et en phase définitive.

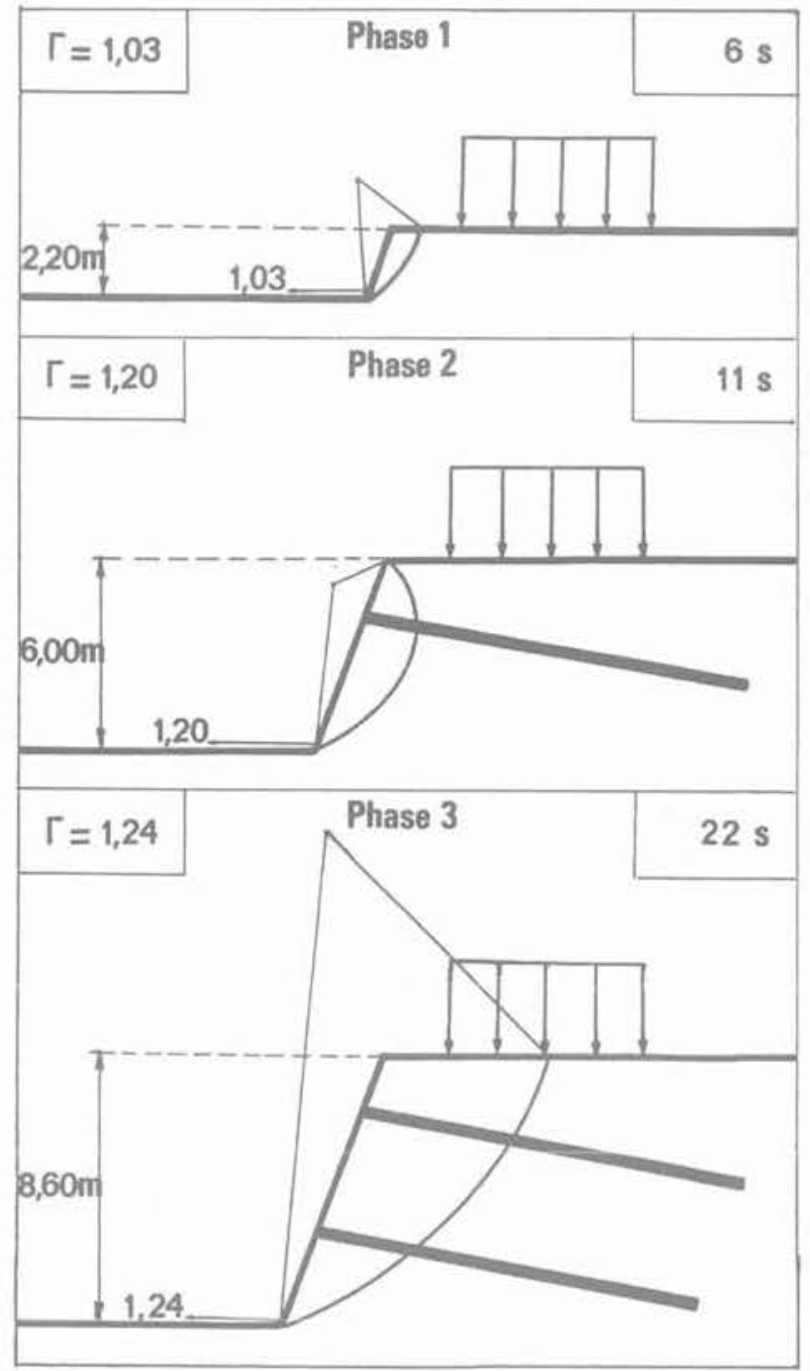

Fig. 17. - Les trois phases de construction. Fig. 17. - The three construction steps.

La solution retenue a été jugée optimale dans un certain contexte technique et économique. Dans un contexte différent elle serait totalement autre.

\section{CONCLUSION}

La méthode de calcul présentée a le double avantage d'être mécaniquement rigoureuse et numériquement extrêmement rapide. En matière de sécurité, sa philosophie est beaucoup mieux adaptée au cas des 
ouvrages renforcés que la conception ancienne développée pour les ouvrages homogènes. La structure informatique du logiciel correspondant (mode conversationnel) permet à l'utilisateur d'exploiter facilement et systématiquement les informations fournies. Grâce à cette souplesse d'utilisation, il peut être utilisé à des fins diverses :

- assistance à la conception et au dimensionnement des ouvrages ;

- optimisation d'un schéma de renforcement selon des critères donnés ;

- contrôle de la stabilité d'un ouvrage existant ;

- étude de la stabilité des phases de construction :

- interprétations de résultats expérimentaux ;

- recherche (études paramétriques, innovations techniques, etc.).

Enfin, la prise en compte des données plus complexes (couches de sol, écoulement, flexion et cisaillement, etc.) ne pose pas de problème particulier dans le cadre de la théorie du calcul à la rupture. La version actuelle du logiciel sera prochainement complétée en conséquence.

\section{BIBLIOGRAPHIE}

ANTHOINE A. (1989), Mixed modelling of reinforced soils within the framework of the yield design theory. Computers and Geotechnics 7. pp. 67-82.

BLONDEAU F., CHRISTIANSEN M., GUILLOUX A., SCHLOSSER F. (1984), Talren : méthode de calcul des ouvrages en sols renforcés. Coll. Int.
Renf. des sols en place, Paris, Presses de I'E.N.P.C., pp. 219-224.

DE BUHAN P., SALENÇON J. (1987), Analyse de la stabilité des ouvrages en sols renforcés par une méthode d'homogénéisation. Revue Française de Géotechnique 41, pp. 22-43.

CHEN W.F. (1975), Chap. IX Stability of slopes. Limit analysis and soil plasticity. Elsevier, Amsterdam, pp. 399-445.

DELMAS Ph., BERCHE J.C., CARTIER G., ABDELHEDI A. (1986), Une nouvelle méthode de dimensionnement du clouage des pentes : programme PROSPER. Bull. Liaison Labo. P. et Ch. 141 , pp. 57-66.

JURAN I., SHAFFIE S., SCHLOSSER F. (1985), Les soutènements par clouage. Etude sur modèles numériques. C.R. XI Cong. Int. Méc. Sols, San Francisco, pp. 1713-1716.

LESHCHINSKY D., REINSCHMIDT A.J. (1985), Stability of membrane reinforced slopes. Jl. of Geotech. Eng., A.S.C.E., vol. 111, n 1, pp. 1285-1300.

PASTOR J., TURGEMAN S., CISS A. (1986), Calculation of limit loads of structures in soils with metal reinforcement. Proc. European Conference on Numerical Methods in Geomechanics, Stuttgart.

SALENÇON J. (1983), Calcul à la rupture et analyse limite. Presses de l'E.N.P.C., Paris.

SALENCON J (1989), An introduction to the theory of yield design and its application to soil mechanics. A paraître.

SIAD L. (1987), Analyse de stabilité des ouvrages en terre armée par une méthode d'homogénéisation. Thèse de doctorat E.N.C.P., Paris.

STARS, un nouveau logiciel pour compatibles $P C$ calcule en quelques secondes le dimensionnement à la rupture des ouvrages en sols renforcés.

STARS, un logiciel développé au laboratoire de mécanique des solides (laboratoire commun E.P. - E.N.P.C. - E.N.S.M.P.) par A. ANTHOINE, P. de BUHAN et J. SALENÇON

En vente aux Presses de l'Ecole Nationale des Ponts et Chaussées dans les prochaines semaines 28, rue des Saints-Pères - 75007 Paris (France) 